\title{
Die unbekannte dritte Dimension: Geländehöhen, Gewässertiefen und Dynamik österreichischer Donaulandschaften vor der Regulierung
}

\author{
Severin Hohensinner · Mathias Jungwirth
}

Online publiziert: 13. Juli 2016

(C) Der/die Autor(en) 2016 . Dieser Artikel ist eine Open-Access-Publikation.

Zusammenfassung Flusslandschaften sind multidimensionale, durch vielfältige räumlich-zeitliche Wechselbeziehungen geprägte Ökosysteme. Longitudinale Interaktionen entlang des Flusskontinuums sowie laterale Austauschprozesse zwischen Fluss und Augebiet stehen bei Restaurationsprojekten zumeist im Vordergrund. Vertikale Wechselwirkungen, wie zum Beispiel zwischen Wasserlebensraum und Flusssohle (hyporheisches Interstitial) oder zwischen Grundwasser und terrestrischen Habitaten, werden oft erst dann in die Planungen miteinbezogen, wenn die Beeinträchtigungen ökologischer Funktionen und menschlicher Nutzungen bereits offensichtlich sind. Dies liegt zum Teil auch daran, dass für die Zeit vor der Regulierung kaum fundierte Grundlagendaten über die Ausformung unserer Flusslandschaften in vertikaler Dimension vorliegen, an denen man sich bei Planungen orientieren könnte.

Ergebnisse mehrerer Forschungsprojekte aus den letzten Jahren gewähren neue Einblicke in die dreidimensionale hydromorphologische Ausprägung der österreichischen Donaulandschaften zu Beginn und Mitte des 19. Jahrhunderts. Sie erlauben eine konkrete höhenmäßige Einstufung verschiedener, ökomorphologisch relevanter $\mathrm{Ge}$ ländezonen innerhalb des Augebiets

DI Dr. S. Hohensinner $(\bowtie) \cdot$ em.o.Univ.-Prof Dr. M. Jungwirth Department für

Wasser-Atmosphäre-Umwelt, Institut für Hydrobiologie und Gewässermanagement, Universität für Bodenkultur Wien, Max-Emanuel-Straße 17, 1180 Wien, Österreich

E-Mail:

severin.hohensinner@boku.ac.at

em.o.Univ.-Prof Dr. M. Jungwirth E-Mail: mathias.jungwirth@boku.ac.at in Bezug zu charakteristischen Wasserständen der Donau. Jede dieser Zonen entspricht einem bestimmten morphologischen Entwicklungsstadium und erfüllt unterschiedliche ökologische Funktionen. Rekonstruktionen der Flurabstände innerhalb des neuzeitlichen, bis zu 500 Jahre alten Augebiets zeigen, dass diese bei Mittelwasser mit durchschnittlich rund 1,6-1,9 m relativ gering waren. Im Vergleich dazu liegen heute die Flurabstände bei rund 3 m oder darüber. Der Wasserkörper der Donau war ehemals nicht nur viel breiter, sondern auch wesentlich flacher ausgebildet als heute. So nahmen bis zu einem Meter tiefe Flachwasserzonen bei sommerlichem Mittelwasser ehemals ca. 8,1 ha pro $\mathrm{km}$ Luftlinie ein, während sie aktuell nur mehr bei 1,2 ha liegen. Dies entspricht einem Rückgang um $86 \%$. Dabei ist noch nicht berücksichtigt, dass es ehemals auch zahlreiche durchströmte Nebenarme mit vergleichbaren Habitaten gab, die heute nicht mehr existieren.

Besonders interessante Einblicke ergeben sich bezüglich der historischen Umlagerungsdynamik der Donau. Im Zuge von avulsiven Laufverkürzungen von Donauhauptarmen wurden zwischen 1812 und 1817 im Mittel jährlich 3 Millionen $\mathrm{m}^{3}$ an Material erodiert und 2,6 Millionen $\mathrm{m}^{3}$ innerhalb desselben Augebiets wieder abgelagert. Rund 0,4 Millionen $\mathrm{m}^{3}$ wurden durchschnittlich jedes Jahr aus dem System ausgetragen und weiter flussabwärts transportiert. Eine derart intensive Umlagerungsdynamik ereignete sich jedoch innerhalb eines bestimmten Flussabschnitts nicht permanent. In den Folgejahren reduzierten sich die erodierten Volumina erheblich, während die Ablagerungen leicht überwogen. Die intensive morphologische Dynamik spiegelte sich auch in signifikanten Absenkungen und Hebungen der Wasser- bzw. Grundwasserspiegellagen innerhalb des Augebiets wider.
Trotz der umfassenden Veränderungen der Flusslandschaft blieben diese in Summe jedoch annähernd ausgeglichen. Auch andere untersuchte Parameter deuten darauf hin, dass sich die hydromorphologische Ausformung von Aulandschaften über größere Flächen und längerfristig betrachtet in einem dynamischen Gleichgewicht oder quasistationären Zustand („steady state“) befanden.

Nicht nur an der Donau, sondern entlang der meisten größeren Flüsse haben die Augebiete heute wesentlich höhere Geländelagen in Relation zum Wasser- bzw. Grundwasserspiegel, als dies vor der Regulierung der Fall war. Eintiefung der Flusssohle einerseits und durch Ablagerung von Sedimenten bei Hochwässern stetig auflandende Augebiete andererseits führen zu einer immer stärkeren vertikalen Entkoppelung der Wasser- und Landlebensräume in Fluss-Auen-Systemen. Die Flurabstände vergrößern sich und die ehemals nassen, feuchten oder frischen Standorte der Weichen Au gehen sukzessive verloren. Die dargestellten Beispiele aus der Geschichte der österreichischen Donau zeigen, dass eine flusstypische - und im Falle der Donau hohe - Umlagerungsintensität, durch die große Anteile der aquatischen und terrestrischen Habitate in vergleichsweise kurzen Zeiträumen regeneriert und verjüngt wurden, als Schlüssel zur Aufrechterhaltung eines heterogenen Habitatkomplexes anzusehen ist.

The unknown third dimension: terrain elevations, water depths and fluvial dynamics of Austrian Danube river landscapes prior to regulation

Abstract River landscapes are multidimensional ecosystems characterized by diverse spatio-temporal interrelations. Longitudinal interactions along the river continuum and lateral ex- 
change processes between river and floodplain are common focal points in river restoration projects. Vertical interactions, such as between aquatic habitats and river bottom (hyporheic interstitial) or between groundwater and terrestrial habitats, are often only addressed in planning schemes, if the impairments of ecological functions and human uses are already evident. This partly reflects the dearth of wellfounded basic data on the configuration of river landscapes prior to regulation in the vertical dimension. Consequently, only few reference data are available for designing adapted restoration measures.

Several research projects in recent years have yielded new insights into the three-dimensional hydromorphological configuration of Austrian Danube landscapes in the early and mid-19th century. They enable the height of different ecomorphologically significant terrain zones within the floodplain to be specified in respect to characteristic Danube water levels. Each of these zones corresponds to a particular morphological stage of development and fulfills specific ecological functions. Reconstructions of the groundwater tables at mean water level within the modern, up to 500-years-old floodplain show that these were on average only 1.6-1.9 $\mathrm{m}$ below the terrain surface. In comparison, today the groundwater table depths amount to c. $3 \mathrm{~m}$ or more. The water bodies of the Danube were formerly not only much broader, but also much shallower. Accordingly, shallow, up to 1-meter-deep water zones originally amounted to c. 8.1 ha per $\mathrm{km}$ linear distance at summer mean water level. In contrast, the current value is 1.2 ha, representing an $86 \%$ decrease. This calculation omits the formerly numerous lotic side arms with comparable habitats that no longer exist today.

Particularly interesting insights were obtained regarding the historical morphological dynamics of the Danube. In the course of avulsive shortenings of Danube main arms, an average 3 million $\mathrm{m}^{3}$ of sediments were eroded annually between 1812 and 1817, and 2.6 million $\mathrm{m}^{3}$ were redeposited in the same floodplain. About 0.4 million $\mathrm{m}^{3}$ were discharged each year from the system and transported further downstream. Importantly, such massive turnover processes did not occur permanently within a particular river section. In subsequent years, the eroded volumes dropped significantly, with deposition clearly exceeding erosion. The intensive morphological dynamics were reflected in significant drawdowns and uplifts of the water and groundwater levels within the floodplain. Despite the extensive changes in the river landscape, in total these hydrodynamics remained roughly balanced. Other examined parameters indicate that viewed over larger areas and longer time periods - the hydromorphological configuration of the river landscape remained in dynamic equilibrium or a type of steady state.

Today, along the Danube River and most other major rivers, floodplains show much higher terrain levels in relation to the water or groundwater level than prior to regulation. Riverbed deepening, combined with constantly increasing levels of the floodplain terrain due to flood-related sediment deposition, led to a progressing vertical decoupling of aquatic and terrestrial habitats in the river-floodplain systems. Depths of the groundwater table relative to the terrain surface increased and formerly wet, moist or fresh sites of softwood forests were successively lost. The examples from the history of the Austrian Danube River illustrate that a river-typical - and in the case of the Danube River high - intensity of fluvial dynamics is crucial in maintaining a heterogeneous habitat complex. These processes regenerate and rejuvenate large parts of the aquatic and terrestrial habitats in relatively short periods of time.

\section{Einleitung}

Im Rahmen der Diskussion und Planung von Restaurationsprojekten an Fließgewässern standen in den letzten Jahren neben der Wiederherstellung leitbildgerechter aquatischer Habitate und der Initiierung dynamischer Umlagerungsprozesse vermehrt auch Überlegungen bezüglich der dreidimensionalen Ausformung naturnaher Flusslandschaften im Zentrum des Interesses. Aufgrund der voranschreitenden Sohleintiefung der Donau im Nationalpark Donau-Auen stellt die Stabilisierung und optimalerweise sogar leichte Anhebung der Sohl- und Wasserspiegellagen die aus auenökologischer Sicht wichtigste Maßnahme des „Flussbaulichen Gesamtprojekts Donau östlich von Wien“ (FGP) dar (Reckendorfer et al. 2005). Auch an anderen
Donauabschnitten oder größeren $\mathrm{Zu}$ bringern wurde bei der Planung von Restaurationsmaßnahmen verstärkt auf eine ökologisch adäquate („ursprüngliche“) Höhenlage des Wasserspiegels oder der Geländeoberkante im Augebiet geachtet. So zum Beispiel im Zuge des LIFE+ Projekts „Flusslandschaft Enns" flussauf des Gesäuses (Muhar et al. 2011) oder zuletzt auch in einem bisher in Österreich noch nie umgesetzten Umfang am Mündungsabschnitt der Traisen (LIFE+ Traisen; Jungwirth et al. 2005; Schmalfuß und Eberstaller 2010). Gründe für die aktuelle Fokussierung auf diese flussmorphologischen Aspekte sind einerseits die Regulierungen im 19. Jahrhundert, wodurch sich die stark eingeengten Fließgewässer eintieften, andererseits die Errichtung von Geschieberückhalteanlagen und Stauhaltungen an alpinen Zubringern im 20. Jahrhundert. Verringerter Feststofftransport, insbesondere des Geschiebes, gepaart mit anhaltender Ablagerung von Feinsedimenten bei Hochwässern in Augebieten führten in vielen Flusslandschaften zu einer zunehmenden Entkoppelung der Höhenniveaus von Wasser- und Landlebensräumen (Jungwirth et al. 2014). Neben dem Trockenfallen von Augewässern und dem Verlust semiaquatischer Habitate macht sich dies primär in zunehmend vergrößerten Flurabständen, dem Höhenunterschied zwischen Geländeoberkante und Grundwasserspiegel, im Augebiet bemerkbar.

Damit verringern sich auch laufend potenzielle Standorte für nasse, feuchte oder frische Auwaldgesellschaften, die dadurch forstwirtschaftlich höherwertig genutzt oder überhaupt einer anderen Nutzungsform zugeführt werden können (Hein et al. 2014; Drescher et al. submitted). Weichholz-Auwälder werden daher basierend auf der FaunaFlora-Habitat-Richtlinie als prioritäre Schutzgüter (Lebensraumtyp „Restbestände von Erlen- und Eschenwäldern an Fließgewässern“) ausgewiesen, für deren Erhalt und Förderung der jeweilige Mitgliedsstaat eine besondere Verantwortung trägt (Natura 2000, FFH-Richtlinie, 92/43/EWG). Im Mündungsabschnitt der Traisen soll dies zum Beispiel durch eine großflächige Absenkung des Vorlandes entlang des neuen, rund 9,5 km langen Flusslaufes bewirkt werden (Haidvogl und Eberstaller 2010). Auch die Umsetzung der EU-Wasserrahmenrichtlinie 2000/60/EG, die als Bewertungsbasis 
den „sehr guten ökologischen Zustand“ vorsieht, in dem auch typspezifische, hydromorphologische Referenzbedingungen Eingang finden, erfordert es in degradierten Flussabschnitten, die dreidimensionale Ausformung von FlussAuen-Ökosystemen miteinzubeziehen (EU 2000). Andernfalls werden die geforderten Ziele bezüglich der biologischen Qualitätskomponenten (,guter ökologischer Zustand“) nicht zu erreichen sein. Jedoch liegt der Fokus heute zumeist nur auf den Fließgewässern selbst, während die angrenzenden Auensysteme kaum berücksichtigt werden (Graf et al. 2013). Eine Ausnahme stellt der in Österreich entwickelte $\mathrm{Au}$ en-Index dar, der als leitbildgemäßes Bewertungsschema unterschiedliche Indikatorgruppen (Fische, Amphibien, Weichtiere, Libellen, Köcherfliegen) innerhalb der gesamten Flusslandschaft miteinbezieht (Chovanec et al. 2005).

Mit Ausnahme weniger Studien zur Auswertung historischer Pegeldaten gab es bis vor rund zehn Jahren keine Untersuchungen zur ehemaligen dreidimensionalen Charakteristik österreichischer Donaulandschaften. Wer nicht selbst historische Quellen auswertete, war auf wenig fundierte Vermutungen angewiesen. Erst im Zuge mehrerer Forschungsprojekte (FWF, proVISION, Jubiläumsfonds der Stadt Wien etc.) wurden in den letzten Jahren diesbezüglich zahlreiche neue Erkenntnisse gewonnen. Der vorliegende Artikel versteht sich als Beitrag dazu, Kenngrößen bezüglich der vertikalen hydromorphologischen Ausformung der österreichischen Donaulandschaften zugänglich zu machen. Der Entstehungsgeschichte der präsentierten Ergebnisse im Rahmen unterschiedlichster Forschungsprojekte geschuldet, handelt es sich dabei nicht um eine systematische Untersuchung entlang der gesamten österreichischen Donau. Vielmehr sind es beispielhafte Auswertungen, die interessante Einblicke in die ehemalige Charakteristik der Flusslandschaft vor der systematischen Regulierung im späten 19. Jahrhundert erlauben. Dennoch können einige Kenngrößen vermutlich generell auch auf die gesamte Donau umgelegt werden, da sie unabhängig voneinander mit geringen Abweichungen für unterschiedliche Donauabschnitte nachgewiesen werden konnten. $\mathrm{Zu}$ berücksichtigen ist jedoch, dass die präsentierten Werte nur für den ehemaligen morphologischen Flusstyp der österreichischen
Donau („gravel-dominated, laterally active anabranching river" nach Nanson und Knighton 1996), nicht aber für andere Fließgewässer gelten, da die hydromorphologische Ausprägung eines Gewässersystems stets ein Produkt seines individuellen Abfluss- und Sedimentregimes ist.

\section{Datenquellen, Methodik und Untersuchungsgebiete}

Bis in die 1980er wurden die flussmorphologischen Charakteristika der österreichischen Donau nur qualitativ anhand von historischen Karten und Ansichten beschrieben. Eine umfassende Quantifizierung von Gewässerstrukturen war nur mit großem zeitlichem Aufwand mithilfe eines Planimeters möglich. Die zunehmende Verbreitung leistungsfähiger Computer ermöglichte es 1986 erstmals, die Entwicklung der Wasser- und Landflächen in der Wiener Donaulandschaft zwischen 1780 und 1981 großflächig zu quantifizieren (Stummer 1986). Einen weiteren Meilenstein setzte der von Peter Mohilla und Franz Michlmayr 1996 herausgegebene „Donauatlas Wien“, in dem historische Karten zwar georeferenziert, aber keine weiteren Auswertungen vorgenommen wurden. Bereits ein Jahr zuvor erfolgte eine systematische Quantifizierung gewässertypischer Flussstrukturen für den Donauabschnitt im Durchbruchstal zwischen Passau und Aschach (Hohensinner 1995). Im Rahmen der genannten Arbeiten wurden jedoch noch keine Auswertungen zu ehemaligen Wassertiefen oder Höhenlagen in Augebieten vorgenommen. Dies erfolgte erstmals im Zuge des FWF-Projektes „Machland 1715 - 1991“ (Projekt-Nr. P14959-B06) für die alluviale Donau zwischen dem Kraftwerk Wallsee-Mitterkirchen und dem Eintritt in den Strudengau bei Ardagger Markt (Strom-km 2094,4-2084; Hohensinner et al. 2004). Dabei erwies es sich als Glücksfall, dass dieser Stromabschnitt (ehemals „Holler“ genannt) aufgrund seiner hohen, die Schifffahrt stark beeinträchtigenden Dynamik bereits sehr früh genau vermessen wurde. Ein von Ing. Friderich (Friedrich) 1812 erstellter Lageplan sowie ein ergänzender Längenschnitt beinhalten neben zahlreichen flussmorphologischen Informationen auch Höhenkoten der Geländeoberkante, Wassertiefen entlang des Stromstrichs und Wasserspiegellagen bei Niederwasser, Mittelwasser und 3- bis 5-jährlichem Hochwasser. Wie damals üblich wurden die Höhen nicht wie heute auf eine niedriger liegende, sondern auf eine höher liegende Vergleichsebene (Nullebene) bezogen. Mangels eines überregionalen Höhenbezugssystems wurden im Flussbau lokal oder regional gültige Nullebenen herangezogen. Damit ist es zwar möglich, relative Höhenunterschiede, wie z. B. zwischen Geländeoberkante und Wasserspiegel, zu berechnen, nicht aber die absolute Höhenlage im heute gebräuchlichen Bezugssystem (m ü. A.) $\mathrm{zu}$ bestimmen. Der lokale Nullpunkt der Holler-Vermessung des Jahres 1812 konnte eindeutig bestimmt und neu eingemessen werden, wodurch nun ein direkter Vergleich mit den aktuellen Verhältnissen möglich ist (der Höhenfixpunkt existiert seit der Absiedelung der Ortschaft Hütting im Zuge des Hochwasserschutzprojektes Machlanddamm 2006 nicht mehr). Da im Operat von Ing. Friderich neben dem lokalen System auch das regionale Höhensystem eingetragen wurde, konnten auf diese Weise auch die Höhendaten der um 1817 bis 1818 erfolgten „Allgemeinen Donau-Aufnahme“ der oberösterreichischen Donau rückberechnet und in das heutige Höhensystem übertragen werden (von dieser ersten genauen Vermessung der oö. Donau liegen nur mehr Fragmente vor; OÖLA, Stromkarten-Mappen). Beinahe zeitgleich wurde 1816 bis 1817 von Christophorus de Lorenzo die „Nieder Oesterreichische Donau-Stromkarte“ erstellt, die unter anderem Informationen zu Fixpunkten, Wasserspiegelgefälle, Uferhöhen, Fließgeschwindigkeiten und Regulierungsbauwerken enthält (Abb. 1). In diesem Vermessungsoperat sind alle Höhendaten auf die Wasserspiegellage beim sogenannten Nullwasserstand (Pegelnullpunkt) bezogen, ebenso die Höhendifferenz zwischen den Fixpunkten.

Im ersten Schritt wurden die Höhenkoten für charakteristische Wasserspiegellagen und Höhenzonen des Augebietes im Machland 1812 ausgelesen und bilanziert (unbewachsene Kiesbänke, junge Anlandungsflächen und Uferzonen, Hauptniveaufläche des Augebietes; Hohensinner et al. 2004). Diese Informationen wurden mit einem umfangreichen Planungsoperat von August Höchsmann (1848) abgeglichen, der das östliche Machland um ca. 1831 anhand eines Lageplans und eines ausführlichen technischen Berichts be- 


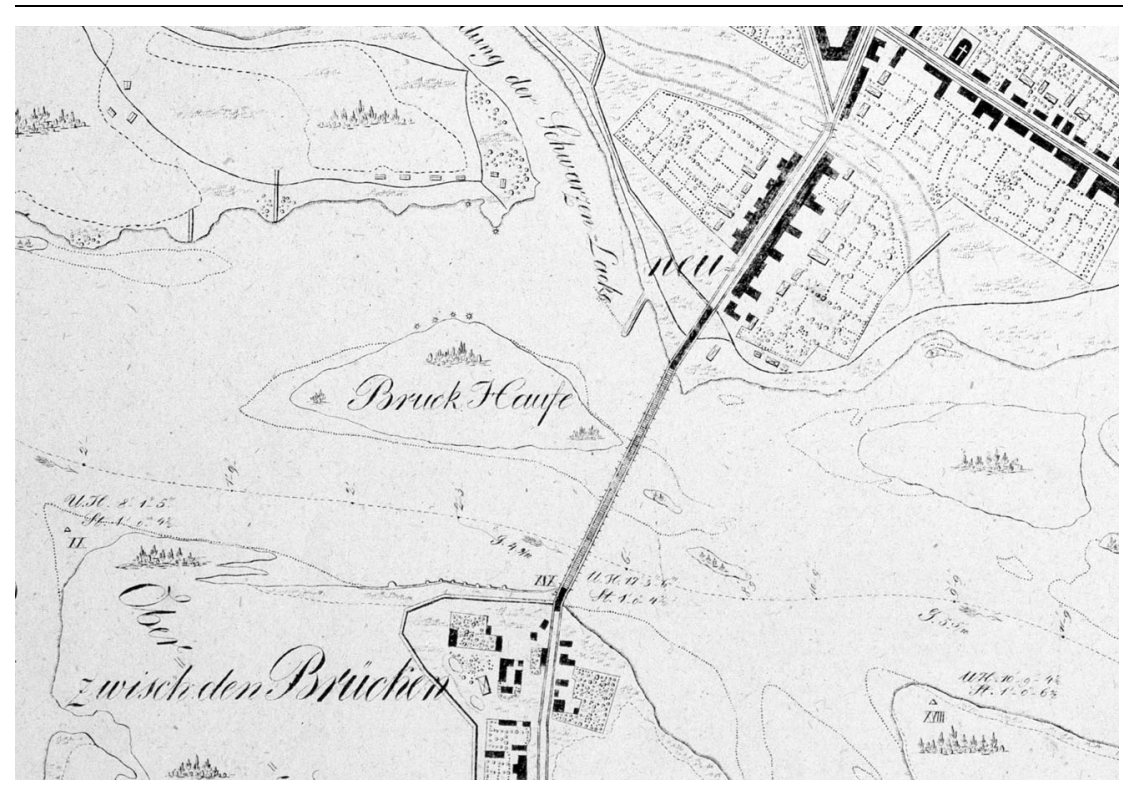

Abb. 1 Ausschnitt aus der der „Nieder Oesterreichischen Donau-Stromkarte“ von Christophorus de Lorenzo aufgenommen 1816 bis 1817. Der Ausschnitt zeigt die Große Taborbrücke mit dem Floridsdorfer Spitz im Norden und der Ortschaft Zwischenbrücken im Süden. Eingetragen sind Uferhöhen, Wasserspiegelgefälle, Wassertiefen und Fließgeschwindigkeiten (NÖ. Landesbibliothek, B II 82)

schrieb. Darauf aufbauend wurde unter Zuhilfenahme zahlreicher weiterer historischer Quellen und aktueller Geodaten ein digitales Geländemodell (DGM) der Flusslandschaft von 1812 mittels Autodesk Land Desktop erstellt, das mit einem aktuellen DGM verschnitten werden konnte. Damit konnten erstmals flächendeckend dreidimensionale Analysen einer historischen Donaulandschaft, eine 2D-tiefengemittelte hydrodynamische Simulation mittels River 2D und die Berechnung von Massendifferenzen zum aktuellen Zustand vorgenommen werden (Hohensinner 2008). Im weiteren Schritt wurden vom gleichen Flussabschnitt vereinfachte Geländemodelle der Situationen in den Jahren 1817 und 1821 (mit besseren Sohldaten) erstellt, um Massenbilanzen für kurzfristige Umlagerungsprozesse berechnen zu können.

All diese Arbeiten hatten den Nachteil, dass die zugrunde liegenden Geländemodelle auf vergleichsweise wenigen Höhenkoten beruhten. Eine wesentlich bessere Grundlage für dreidimensionale Analysen bietet die „Hydrotechnische Vermessung“ der Wiener Donaulandschaft im Jahr 1849, aufgenommen bei sommerlichem Niederwasser unter der Leitung von Valentin von Streffleur. Während das ursprüngliche Operat verschollen ist, existiert heute noch ein plastisches Geländemodell im Tech- nischen Museum Wien, das von Carl Drobny mit einem Isohypsenabstand von 0,2 Klafter $(0,38 \mathrm{~m})$ erstellt wurde. Vermutlich in den 1970ern rekonstruierte Karl Killian daraus den „Lage- und Wien 1849“, welcher mittels ca. 250 Höhenpunkten neu kalibriert wurde und heute im Archiv der MA 29 - Baugrundkataster liegt. Dieser Plan sowie andere Vermessungen der Donausohle und Wasserspiegellagen 1846/47, die vermutlich ebenso auf Valentin von Streffleur zurückgehen, bildeten die Grundlage für ein digitales Geländemodell, das von Mathew Herrnegger mittels ESRI ArcGIS für den Bereich zwischen Strom-km 1933,5 und 1921,6 erstellt wurde (Abb. 2; Herrnegger 2007). In Kombination mit eigenen Auswertungen der Pegelstände an der Großen Taborbrücke (siehe Abb. 1) zwischen 1828 und 1849 können sehr genaue Aussagen über ehemalige Flurabstände oder Überflutungshöhen getroffen werden (Hohensinner et al. 2008).

Mittlerweile wurde u. a. basierend auf der Vermessung von Lorenzo 1816 bis 1817 auch ein DGM für den Bereich der Wiener Lobau analog zur Methodik im Machland mittels ArcGIS erstellt und mit HEC-RAS eine 1D-hydrodynamische Simulation vorgenommen. Das Projekt im Auftrag der MA 45 Wiener Gewässer bzw. WasserCluster Schichtenplan des Donaugeländes bei
Lunz - Biologische Station GmbH ermöglichte detaillierte Einblicke in die ehemalige hydromorphologische Charakteristik unterschiedlicher Typen von Augewässern (Wassertiefen, Fließgeschwindigkeiten etc.; Hohensinner und Trautwein 2013). Weitere Auswertungen historischer Quellen mit Informationen zur vertikalen Ausformung der Wiener Donau-Auen wurden zuletzt im Rahmen des FWF-Projekts „Umweltgeschichte der Wiener Donau 1500 1890“ (ENVIEDAN, Projekt-Nr. P22265G18; Hohensinner et al. 2013a, b) und „Genug Holz für Stadt und Fluss? Wiens Holzressourcen in dynamischen Donau-Auen“ (Jubiläumsfonds der Stadt Wien für die ÖAW; Hohensinner et al. 2013c) durchgeführt.

Die zugrunde liegende Methodik und die Ergebnisse der genannten Projekte erlauben zwar keine systematischen, für die gesamte österreichische Donau gültigen Aussagen hinsichtlich der vertikalen Zonierung der Aulandschaften und der Wasserkörper, bieten aber historisch belegbare Grundlagen für die Diskussion und Planung von Restaurationsprojekten aus dem Blickwinkel unterschiedlicher damit befasster Fachdisziplinen.

\section{Ergebnisse}

\subsection{Generelle Charakteristik alluvialer Donauabschnitte}

Vor Beginn der Regulierung in der ersten Hälfte des 19. Jahrhunderts wiesen die alluvialen Abschnitte der österreichischen Donau sowohl Elemente eines verzweigten als auch eines gewundenmäandrierenden Flusstyps auf (Abb. 3). Das Gerinnesystem umfasste zumeist ein oder zwei dominante Flussarme (Hauptstrom) sowie zahlreiche Nebenund Altarme unterschiedlichster Breite. Großflächige Kiesbänke an den Gleitufern (point bars), aber auch Kiesinseln in der Flussmitte (mid-channel bars) waren charakteristische Elemente in den breiteren Armen. Dementsprechend sind Österreichs alluviale Donauabschnitte im Zustand vor der Regulierung nach dem Klassifikationssystem von Yalin und da Silva (2001) generell als verzweigter Flusstyp einzustufen (Abb. 4).

Zahlreiche kleine, dynamische Inseln waren ebenso charakteristisch wie großflächige, stabilere Inseln, die zum Teil gleiche Geländelagen wie das angrenzende Augebiet aufwiesen. Einzel- 


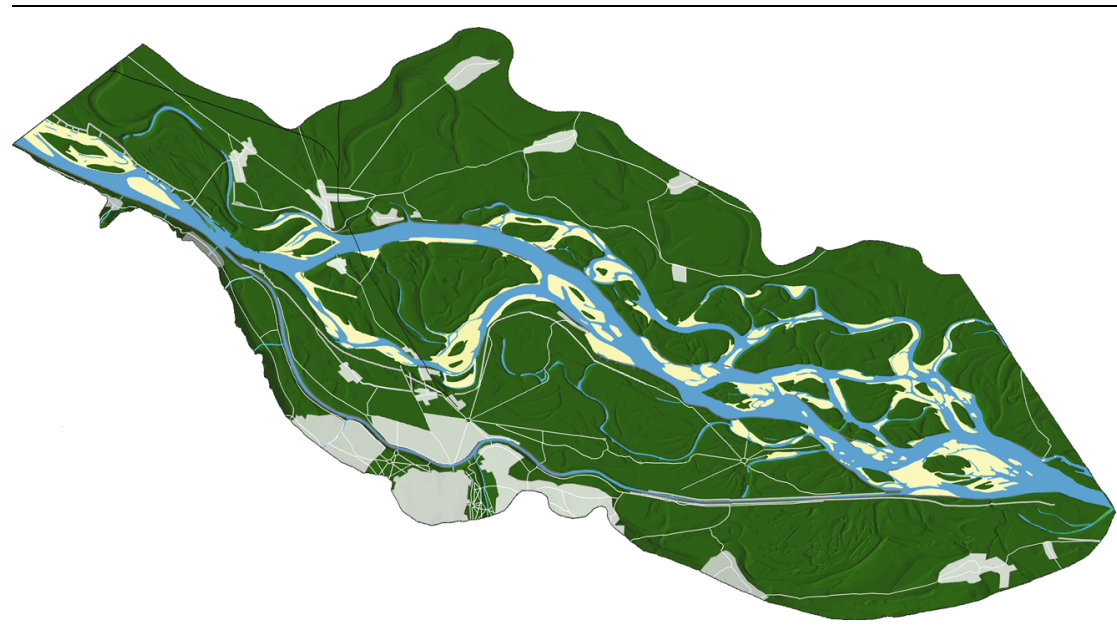

Abb. 2 Digitales Geländemodell 1849 der Wiener Donau-Auen (Kuchelau -Albern), erstellt auf Basis historischer Vermessungsdaten (Herrnegger 2007)

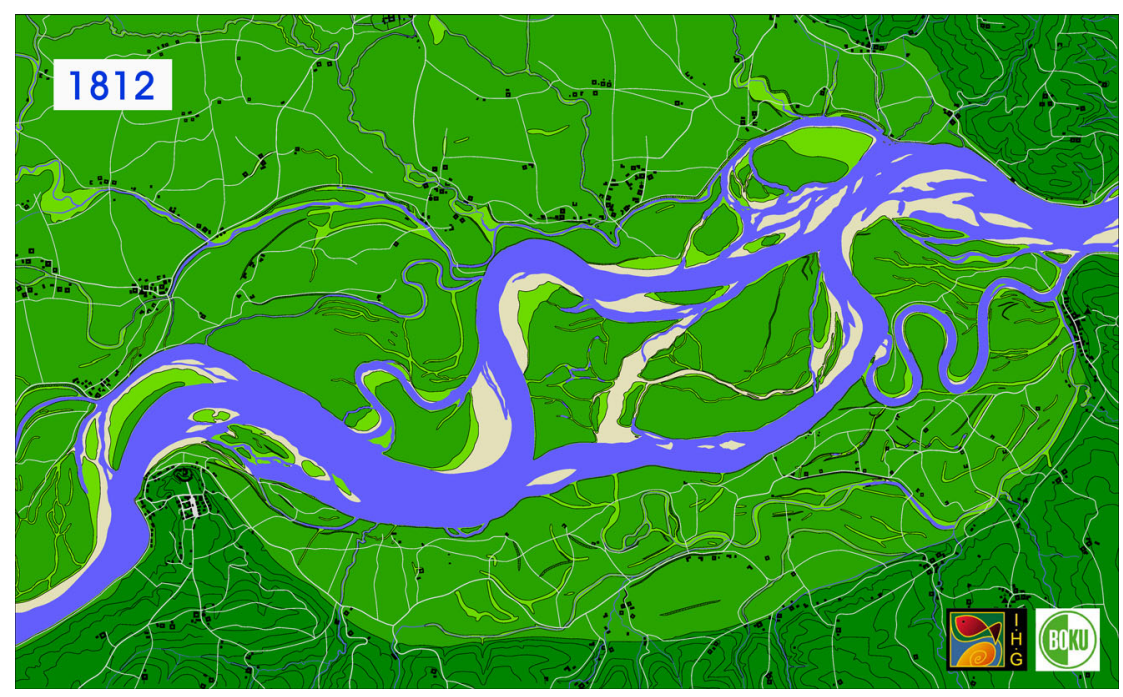

Abb. 3 Flussmorphologischer Zustand der Donaulandschaft im östlichen Machland zwischen Wallsee und Ardagger Markt vor Regulierung im Jahr 1812. Charakteristisch waren ein oder zwei dominante Hauptarme, kleine dynamische und große stabile Inseln (wie der Weidenhaufen in der Bildmitte) sowie verzweigte, gewunden oder sogar mäandrierende Nebenarme (hellgrüne Bereiche: vegetated area below bankfull; Hohensinner 2008)

ne Nebenarme waren stark gewunden oder bildeten zeitweise vollständige Mäander aus. Unter diesem Blickwinkel ist die historische Donau entsprechend dem flussmorphologischen Klassifikationsschema nach Nanson und Knighton (1996) am besten als „gravel-dominated, laterally active anabranching river" oder nach Desloges und Church (1987) als „wandering gravel-bed river“ zu bezeichnen. Gründe für die Entstehung derartiger Flusslandschaften sind neben stark schwankenden $\mathrm{Ab}$ flüssen (alpin geprägtes Regime) und hohen Geschiebeeinstößen (vor 1850 ca. $500.000 \mathrm{~m}^{3} / \mathrm{Jahr}$; Schmautz et al. eines komplexen Gerinnesystems, gekennzeichnet durch allmähliche Laufverlagerungen, aber auch plötzlich auftretende Gerinneverlagerungen (Avulsionen).

Aufgrund des alpin geprägten Feststoffregimes, der individuellen Genese der Gewässer und der unterschiedlichen Überflutungsprozesse, waren diese Systeme durch vielfältige Sedimentationsprozesse gekennzeichnet: laterale Gleitufersedimentation, Geschiebeablagerungen in breiteren Gerinneprofilen (Bankbildungen), Rückstausedimentation, vertikale Sedimentation im Augelände bei Hochwasser etc. Auch vor der Regulierung wiesen die Auböden über den Kiesablagerungen vielfach rund 2,5 m mächtige Sand- und Schlufflagen auf (Höchsmann 1848; Roidtner 1859). Über längere Zeiträume hinweg befinden sich solche Augebiete (medium-energy, non-cohesive, wandering gravel-bed river floodplains vom Typ B2 gemäß Nanson und Croke 1992) jedoch - sofern sich die äußeren Rahmenbedingungen nicht verändern - in einem dynamischen Gleichgewichtszustand von Sedimentation und Erosion.

Ein solches Gleichgewicht setzt neben gleichbleibendem Abflussregime und Sedimenttransport auch weitgehend unveränderte Gefällsverhältnisse voraus, wobei alle drei Komponenten in einem engen ursächlichen Zusammenhang zueinander stehen. Naturgemäß änderte sich das Gefälle in den alluvialen Abschnitten der Donau, je nachdem, ob sie einen längeren, gewundeneren oder einen kürzeren, gestreckteren Lauf ausbildete. So schwankte das Wasserspiegelgefälle zum Beispiel im östlichen Machland im Zeitraum 1775 bis 1817 abhängig vom jeweiligen flussmorphologischen Zustand zwischen $0,47 \%$ und 0,59 \%o (mittleres Gefälle der österreichischen Donau aktuell: 0,43\%o). Als im 19. Jahrhundert an der Donau die ersten genaueren Höhenvermessungen vorgenommen wurden, stellte man jedoch etwas Erstaunliches fest: Das Gefälle der Donau war in den meisten Beckenlagen größer als in den Durchbruchstrecken. Dabei würde man intuitiv eher das Gegenteil erwarten, da die Donau in den Augebieten vielfach stark gewunden war. Dementsprechend betrug das Gefälle direkt flussab des Machlandes im Strudengau nur 0,33\%. Lediglich im Marchfeld wich die Donau von diesem Muster ab. Hier betrug das mittlere Gefälle vor der Regulierung zwischen 
$0,36 \%$ und $0,40 \%$, was vermutlich mit der besonderen Situation der stetigen Absenkung des Wiener Beckens ("Schwechat Tief“) zusammenhängt (Grupe und Payer 2014). Bei näherer Betrachtung werden diese Gefällsverhältnisse aber durchaus erklärbar. Die Sedimenttransportkapazität der Donau war in den kompakteren Gerinnen der Engtäler größer als in den breiteren, verzweigten und daher seichteren Flussarmen der Beckenlandschaften. Um eine im Großen und Ganzen ausgeglichene Transportkapazität entlang des gesamten österreichischen Laufes $\mathrm{zu}$ erreichen, musste sich in den Becken langfristig ein höheres Gefälle als in den Engtälern einstellen (vgl. dazu Schmautz et al. 2002). Andernfalls hätten sich die Becken sukzessive mit Sedimenten gefüllt, was phasenweise durchaus auch der Fall war. Insgesamt gesehen entwickelte sich auf diese Weise nach der letzten Eiszeit bezüglich der Sedimente ein dynamisches Transportgleichgewicht zwischen alluvialen Abschnitten und Durchbruchstrecken.

\subsection{Geländehöhen im Augebiet}

Aus flussmorphologischer und speziell vegetationsökologischer Sicht sind zwei Höhenzonen im Augebiet von besonderem Interesse: die Grenze zwischen offenen Rohböden (Sedimentbänken) und mehrjähriger Vegetation (Gehölze) sowie die Oberkante der Uferböschungen, die zugleich den Übergang zum Hauptniveau des Augebiets bildet. Die Höhenlage beider Niveaus war vor der Regulierung charakteristisch für den jeweiligen Flusstyp und ergab sich aus dem Zusammenspiel von Abflussregime, Sedimenttransport und der lokalen geomorphologischen Situation (z. B. Breite des Tales oder Lage zum nächsten Engtal flussab). Das untere ökomorphologisch relevante Niveau entspricht der Begrenzung des sogenannten aktiven Gerinnes eines Fließgewässers, das aus Wasserflächen und unbewachsenen Sedimentflächen gebildet wird. Entsprechend der Auswertung historischer Quellen lag diese Grenze an der österreichischen Donau generell ungefähr bei 1,7 $\mathrm{m}$ über dem Nullwasserstand. Zu Anfang des 19. Jahrhunderts, als die Pegelnullpunkte der wichtigsten Donaupegel festgelegt wurden, achtete man darauf, dass der Wasserstand - mit Ausnahme von extremen Niederwassersituationen - nie unter den Pegelnullpunkt fiel, um negative Pegel- werte zu vermeiden (Mitis 1835; Pasetti 1862). Nach Auswertung historischer Pegeldaten kann das Nullwasser im frühen 19. Jahrhundert ungefähr mit dem mittleren jährlichen Niederwasserstand (MJNW) gleichgesetzt werden (Hohensinner et al. 2013c). Aufgrund natürlicher Sohlveränderungen und mit zunehmender Regulierung veränderte sich der ursprünglich angedachte enge Zusammenhang zwischen Niederwasser und Pegelnullpunkt. Durch die beginnende Regulierung der Donau und die damit einhergehende Eintiefung der Sohle ergaben sich an einigen Donaupegeln bis in die 1850er vermehrt negative Pegelwerte, weshalb die Pegelnullpunkte im Jahr 1854 neu kalibriert wurden (Wex 1873).

Um die methodischen Unsicherheiten bezüglich des Niederwasserstandes zu umgehen, ist es praktikabler, einen Bezug zum Mittelwasserstand (MW) herzustellen. Dementsprechend lag die Grenze des aktiven Gerinnes bzw. der Gehölzvegetation ehemals $0,4 \mathrm{~m}$ über dem Mittelwasser (Hohensinner et al. 2004). Im Vergleich dazu liegt diese Grenze an der heutigen regulierten Donau mit rund 0,5 m generell etwas höher. Der Grund für diesen Unterschied liegt vermutlich darin, dass der Fluss heute zumeist nur mehr die Hälfte oder ein Drittel seiner ursprünglichen Breite aufweist und die Wasserspiegelschwankungen im engeren Gerinne stärker ausfallen. Die beschriebene Höhenlage von 1,7 m über MJNW bzw. 0,4 m über MW entspricht an der österreichischen Donau ungefähr dem sommerlichen Mittelwasserstand (SMW), also dem mittleren Wasserstand während der Vegetationsperiode. Unter diesem Niveau konnte sich auf offenen Sedimentflächen primär nur annuelle krautige Pioniervegetation entwickeln - für das Aufkommen von Gehölzen war hier die Überflutungshäufigkeit $\mathrm{zu}$ hoch bzw. der Zeitpunkt der Überflutung ungünstig (Drescher und Egger 2013). Gehölzvegetation, zumeist Weidenanflug (Purpurweide, Salix purpurea) oder Deutsche Tamariske (Myricaria germanica) konnte sich hingegen erst über SMW etablieren.

Die hier geschilderte Situation traf nur auf Bereichen an zumindest zeitweise durchströmten Flussarmen zu, in denen An- und Auflandungsprozesse dominierten (wobei ehemals 85 bis $95 \%$ aller Gewässer in den Donau-Auen diesem Typus zugehörten; Hohensinner und Jungwirth 2009). In verlandenden Altarmen mit geringerer Dynamik und feinerem Substrat konnte sich mehrjährige Vegetation hingegen schon auf Mittelwasserniveau (= MJNW $+1,3 \mathrm{~m}$ ) entwickeln. Dies jedoch erst auf Standorten, die älter als ca. 40 Jahre waren; davor dominierten Röhrichtgesellschaften (Drescher und Egger 2013; Hohensinner et al. 2013c). Zu beachten ist beim geschilderten engen Zusammenhang zwischen SMW und der Begrenzung des aktiven Gerinnes jedoch auch, dass es sich hierbei um einen generellen Richtwert handelt. Aufgrund verstärkter Umlagerungsdynamik (Erosion, Anlandung) bei Hochwässern und vor allem menschlicher Eingriffe konnte es zeitweise durchaus auch vorkommen, dass Kiesbänke selbst einen Meter über MW noch keinerlei Gehölzvegetation aufwiesen.

Der Bereich zwischen den beiden ökomorphologisch relevanten Niveaus, also zwischen SMW und der Böschungsoberkante, kann am besten pauschal als „vegetated area below bankfull“ (VABB) beschrieben werden, da diese Zone sehr heterogen strukturiert war (Hohensinner et al. 2011). Darunter fielen Uferböschungen an Prallufern, flache bewachsene Uferzonen an Gleitufern, niedrige bewachsene Inseln sowie Gräben (verlandete Altarme) und Senken im Augebiet (vgl. hellgrüne Bereiche in Abb. 3). All diese Bereiche hatten gemein, dass sie bereits bei gering erhöhter Wasserführung über SMW überströmt bzw. überstaut werden. Je nach Lage in dynamischen Anlandungs- und Auflandungsbereichen oder stabileren Verlandungszonen fernab aktiver Flussarme bildeten sich hier unterschiedliche Typen junger Weichholzgesellschaften (zumeist Weidenauen) aus. Da es sich dabei entlang aktiver Flussarme zumeist um sehr junge Standorte handelte, waren diese von besonderer vegetationsökologischer Bedeutung (Drescher und Egger 2013). Nach oben hin wurden diese von der Böschungsoberkante - gleichbedeutend mit der Hauptniveaufläche des Augebiets - begrenzt.

Diese obere ökomorphologisch bedeutende Grenze entspricht aus gerinnehydraulischer Sicht dem sogenannten bordvollen Wasserstand, also jenem Abfluss, bei dem sich das Wasser gerade noch nicht über die Böschungsoberkante in die Hauptniveaufläche des Augebiets ergießt. Solange der Abfluss eines Gewässers bei einem anwachsenden Hochwasser innerhalb des Ge- 

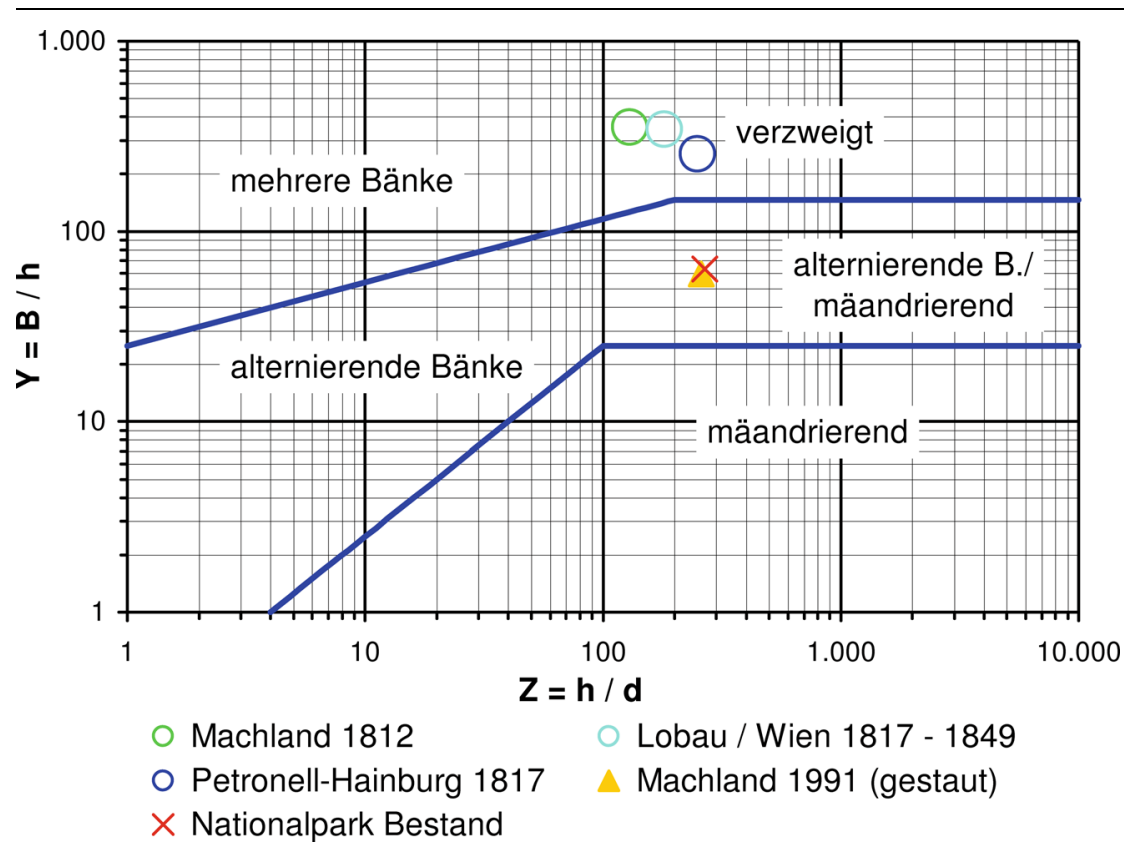

Abb. 4 Flussmorphologische Einstufung alluvialer Donauabschnitte in Österreich vor und nach der Regulierung in Abhängigkeit von der mittleren relativen Abflusstiefe $Z=h / d$ und der relativen Flussbettbreite $\mathrm{Y}=\mathrm{B} / \mathrm{h}$ (nach Yalin und da Silva 2001; $\mathrm{B}=$ bordvolle Breite, $\mathrm{h}=$ bordvolle Tiefe, $\mathrm{d}=$ mittlerer Korndurchmesser $\mathrm{d}_{50}$ ). Zur Einstufung der Situationen vor der Regulierung wurden $B$ und $h$ basierend auf historisch gemessenen Gelände-/Wasserspiegellagen und Geländemodell-Rekonstruktionen ermittelt (Herrnegger 2007; Hohensinner 2008). Für die Korndurchmesser wurden die frühest verfügbaren Daten vom HZB (1937) verwendet, die jedoch aus der Zeit nach der Niederwasserregulierung stammen

rinnes verbleibt, entfaltet er dort die größte Sohlschubspannung (Schleppkraft) und kann durch erosive Prozesse das Gerinne leichter umformen (Leopold und Wolman 1957; Parker et al. 2007). Sobald die Böschungsoberkante überströmt wird und ein Teil des Abflusses das Gerinne verlässt, reduziert sich das Potenzial, das Gerinne zu verändern. Daher wird der bordvolle Abfluss $Q_{b f}$ auch oft mit dem bettbildenden, effektiven oder dominanten Abfluss gleichgesetzt, wobei diese im Grunde genommen aber unterschiedlich definiert sind (für nähere Details dazu siehe Van den Berg 1995; Powell et al. 2006; Klasz et al. 2012). Generell wird für den bordvollen Abfluss in der Literatur eine Hochwasserjährlichkeit von ein bis zwei Jahren angegeben, wobei meist der Mittelwert $\mathrm{HQ}_{1,5}$ als Näherungswert mit hinreichender Genauigkeit betrachtet wird (Wolman und Leopold 1957; Doyle et al. 2007). Erste Auswertungen der historischen Geländelagen anhand eines DGM der Flusslandschaft im Machland 1812 legten jedoch nahe, dass der ehemalige bordvolle Abfluss mit $\mathrm{HQ}_{1}$ eher am unteren Ende des lich geringer war als heute. Das bedeutet, dass die Böschungsoberkanten bzw. Hauptniveauflächen des Augebiets in Relation zum Nullwasserspiegel (MJNW) oder Mittelwasserspiegel (MW) damals tiefer lagen (bzw. im Hinblick auf regulierungsbedingte Eintiefung lagen die Wasserspiegel in Relation zum Auenniveau höher). Die Geländezone der „vegetated area below bankfull“ (VABB) wurde demnach im Mittel zumindest einmal pro Jahr zur Gänze überflutet. Durch den Fließwiderstand (Rauigkeit) der großteils jungen Gehölzvegetation in diesem Bereich verringerte sich die Fließgeschwindigkeit und mittransportierte Schwebstoffe fielen verstärkt aus. Folglich wies diese Geländezone starke An- und Auflandungen auf und entwickelte sich relativ rasch zu höherliegendem Augelände.

Die oberste Geländezone einer Flusslandschaft wird durch das generelle Auenniveau (Hauptniveau des Augebietes) gebildet. Wie bereits erwähnt, wurde es an der Donau vor der Regulierung vermutlich bereits bei einem 1-jährlichen Hochwasser vom Wasser erreicht und in Teilbereichen überflutet. Dabei ist der Übergang von der VABB zum Hauptniveau in einem so stark verzweigten Flusssystem wie dem der ehemaligen Donau nicht eindeutig festzulegen. Anabranched rivers wie die Donau weisen aufgrund der vielfältigen morphodynamischen Prozesse einen sehr hohen Strukturierungsgrad auf, wodurch alleine auf Grundlage von historischen Lageplänen eine eindeutige Zuordnung zu den genannten Geländezonen schwierig ist. Profilaufnahmen der ehemaligen Donaulandschaft zeigen aber, dass die zuvor postulierten großen, stabilen Inseln mit annähernd gleich hohen Geländelagen wie im umgebenden Augebiet als typisches Merkmal eines anabranched rivers durchaus zutreffen. Dadurch lässt sich auch das Hauptniveau des morphologisch älteren Augeländes besser definieren.

Abb. 5 zeigt schematisch die ökomorphologische Höhenzonierung der Donau-Auen im Machland 1812 basierend auf historischen Höhenkoten. Erkennbar ist, dass sich die einzelnen Typen der Geländezonen höhenmäßig überlappen. So konnten tiefliegende Vegetationsflächen (VABB) in Höhenlagen zwischen 1,3 $\mathrm{m}$ und 3,0 $\mathrm{m}$ über MJNW identifiziert werden (im Mittel 1,9 m), während jene des höherliegenden Hauptniveaus vergleichsweise breit gestreut zwischen $2,2 \mathrm{~m}$ und $5,2 \mathrm{~m}$ 


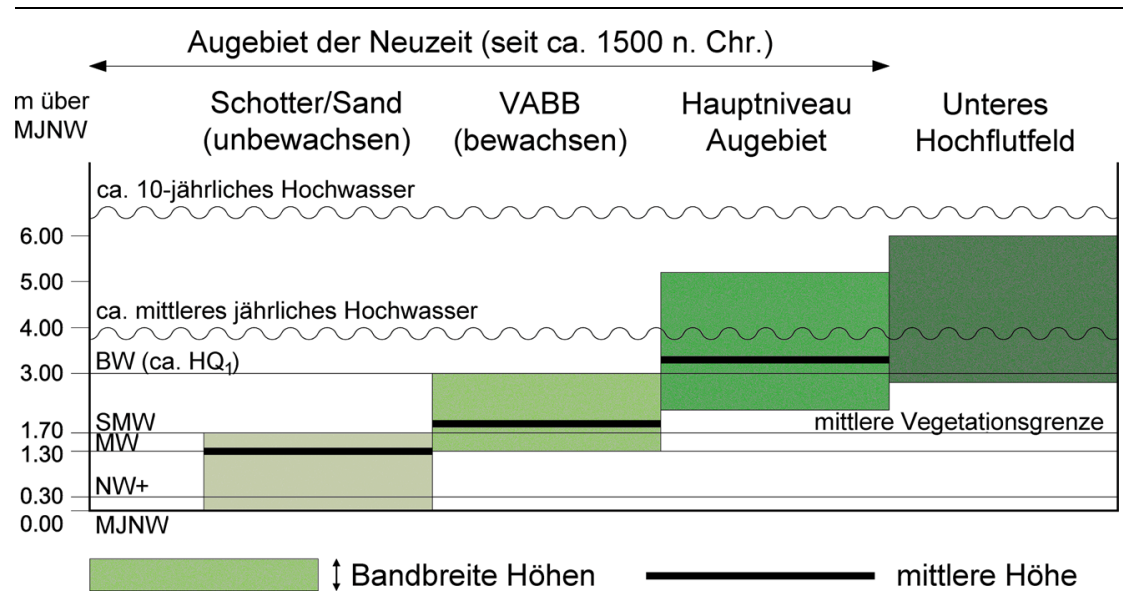

Abb. 5 Schematische Gliederung des Augebietes in charakteristische ökomorphologische Geländezonen im Machland 1812 (MJNW mittleres jährliches Niederwasser, $N W+$ erhöhtes Niederwasser, MW Mittelwasser, SMW sommerliches Mittelwasser, $B W$ bordvoller Wasserstand, $V A B B$ vegetated area below bankfull/tiefliegende Vegetationsflächen; verändertnach Hohensinner etal. 2004, mit Genehmigung von John Wiley \& Sons)

anzutreffen waren (im Mittel 3,3 m über MJNW; um die Werte auf den Mittelwasserstand $\mathrm{zu}$ beziehen, müssen $1,3 \mathrm{~m}$ subtrahiert werden). Die stark variierenden Höhenlagen deuten darauf hin, dass es sich hierbei um einen sehr heterogenen Komplex handelt, der über Jahrhunderte als morphologisches Mosaik verschiedenster Umlagerungsprozesse entstanden ist. Die ältere Flussterrasse des Unteren Hochflutfeldes, die vermutlich während der Römerzeit, spätestens aber im Hochmittelalter entstanden ist, wies noch geringfügig höhere Lagen auf (Hohensinner et al. 2004). Da es sich bei den Donau-Auen im östlichen Machland (ehemaliger „Holler“) um einen ausgesprochen dynamischen Donauabschnitt mit vergleichsweise ,jungem“ Augelände handelte, liegt die Vermutung nahe, dass dadurch auch die Geländelagen generell niedriger waren als in anderen Augebieten (Hohensinner et al. 2011). Der Vergleich zum etwas stabileren Auensystem in Wien und der Lobau scheint das zu bestätigen. Hier lag das Hauptniveau des Augebietes etwas höher zwischen 2,7 $\mathrm{m}$ und 6,7 $\mathrm{m}$ über MJNW (im Mittel 3,8 m bzw. bezogen auf MW im Mittel 2,5 m), wobei in den höchsten Lagen vermutlich Reste älterer Flussterrassen integriert sind (z. B. im Bereich der Leopoldstadt und der Rossau; Hohensinner et al. 2013c).

\subsection{Flurabstände}

Die intakte Verbindung zum Grundwasserkörper, konkret die Distanz zwischen Geländeoberkante und Grundwasserspiegel (Flurabstand), ist für zahlreiche Organismen des Donau-Auenökosystems von zentraler Bedeutung. Daher stellt sich insbesonders die Frage, inwieweit die heutigen Verhältnisse von den ehemaligen Bedingungen abweichen. In Kenntnis der ehemaligen Gelände- und Wasserspiegellagen konnten die Flurabstände im Augebiet vor der Regulierung mittels digitaler Geländemodelle rekonstruiert werden (Hohensinner et al. 2005, 2008). Die Ergebnisse zeigen deutlich, dass die Flurabstände damals erheblich geringer waren als heute; das heißt, dass der Grundwasserspiegel wesentlich näher bei der Geländeoberfläche lag. So betrug der mittlere gewichtete Flurabstand bei Mittelwasser in der flussmorphologisch aktiven Zone der Neuzeit (AZ) im Machland 1812 nur 1,6 m (Medianwert $=1,5 \mathrm{~m}$; vgl. Abb. 6a). Die AZ entspricht dem jüngeren Bereich des postglazialen Augebiets, das unter den klimatisch-hydrologischen Bedingungen der Neuzeit seit ca. 1500 n. Chr. entstanden ist und in dem sich seither dynamische Umlagerungsprozesse zugetragen haben. Dieser Bereich Sicht von besonderem Interesse, da hier noch am ehesten Relikte der ehemaligen Flusslandschaft verblieben sind, während die älteren Bereiche des Augeist heute aus restaurationsökologischer bietes mittlerweile zumeist anderwertig genutzt werden. Die analoge Analyse der Wiener Donau-Auen ergab für das Jahr 1849 mit 1,9 m im Mittel (ebenso im Median) einen geringfügig höheren Wert (Herrnegger 2007; Hohensinner et al. 2008). Dies ist vermutlich einerseits auf die generell etwas höheren Geländelagen bei Wien zurückzuführen, da das Augebiet etwas stabiler und damit älter war als im Machland, und andererseits darauf, dass hier um 1849 bereits zahlreiche Prallufer geschützt und Altarme abgetrennt waren. Dadurch ist um 1849 bereits mit einer beginnenden Verlandung im Augebiet und allgemein größeren Flurabständen zu rechnen. Bezieht man auch das restliche postglaziale Augebiet in die Berechnung mit ein, so ergeben sich naturgemäß etwas höhere Flurabstände: im Machland 1812 im Mittel 1,9 m und in Wien 1849 rund 2,3 m. Aussagen bezüglich der Grundwasserdynamik infolge veränderter Wasserführung der Oberflächengewässer lassen sich anhand der für unterschiedliche Wasserstände rekonstruierten Flurabstände ableiten. Demnach betrug die mittlere Schwankung des Grundwasserspiegels zwischen dem Nullwasserstand (ca. MJNW) und Mittelwasser im Machland 1812 ca. $1,3 \mathrm{~m}$ und bei Wien 1849 ca. 1,5 $\mathrm{m}$ (bezogen auf die AZ).

Für die Vermessung der Flusslandschaft im Machland 1812 und 1817 wurden teilweise die gleichen Fixpunkte herangezogen. Durch die Verschneidung der Wasser- bzw. Grundwasserspiegellagen $\mathrm{zu}$ beiden Zeitpunkten lässt sich abschätzen, inwiefern sich diese durch natürliche Umlagerungsprozesse verändert haben. Zwischen 1812 und 1817 ereignete sich eine weitreichende Umformung der Flusslandschaft, im Zuge derer sich auch die Spiegellagen stark veränderten (siehe dazu Kapitel 3.5. Dynamik und Abb. 12). Demzufolge war beinahe das gesamte postglaziale Auensystem von einer Veränderung der hydrologischen Bedingungen in unterschiedlich starkem Ausmaß betroffen (Abb. 7). Rund $18 \%$ des Augebietes waren von einer starken Absenkung oder Erhöhung der Wasserbzw. Grundwasserspiegellagen um 0,5 bis $1 \mathrm{~m}$ gekennzeichnet und $3 \%$ im Ausmaß von über einem Meter. Wägt man sowohl Absenkungen als auch Erhöhungen gegeneinander ab, so waren diese beinahe vollständig ausgeglichen (theoretisch ergibt sich für das gesamte Auensystem eine Nettoabsenkung der 


\section{Originalarbeit}

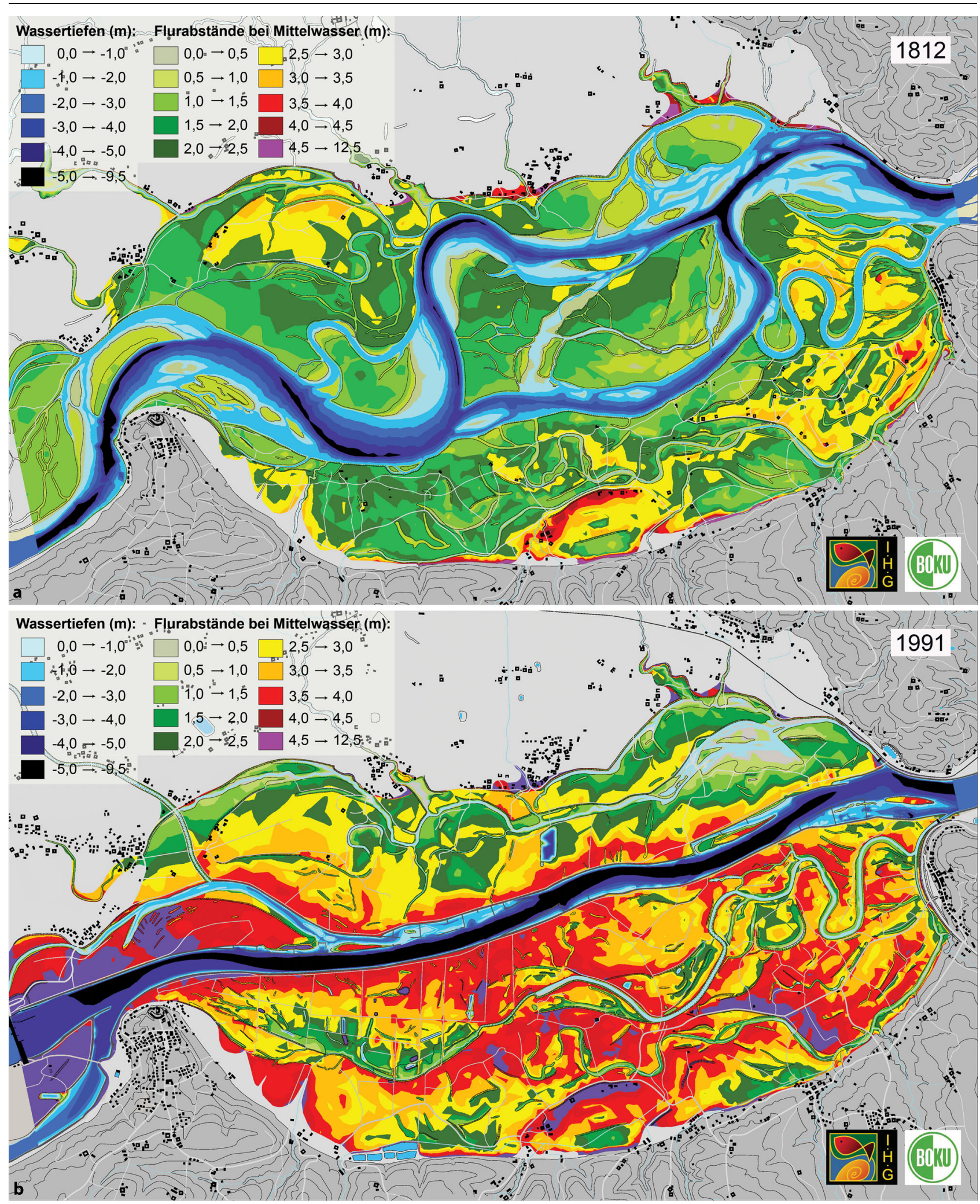

Abb. 6 Flurabstände bei Mittelwasser im gesamten postglazialen Augebiet des östlichen Machlandes: a vor der Regulierung 1812 (oben), Bauernhöfe (schwarze Punkte im Augebiet) waren zumeist auf trockeneren Stellen (orange und rot) angesiedelt; $\mathbf{b}$ nach Regulierung und Einstau 1991 (unten), das Austrocknen des Augebiets ist klar erkennbar (Jungwirth et al. 2014) 


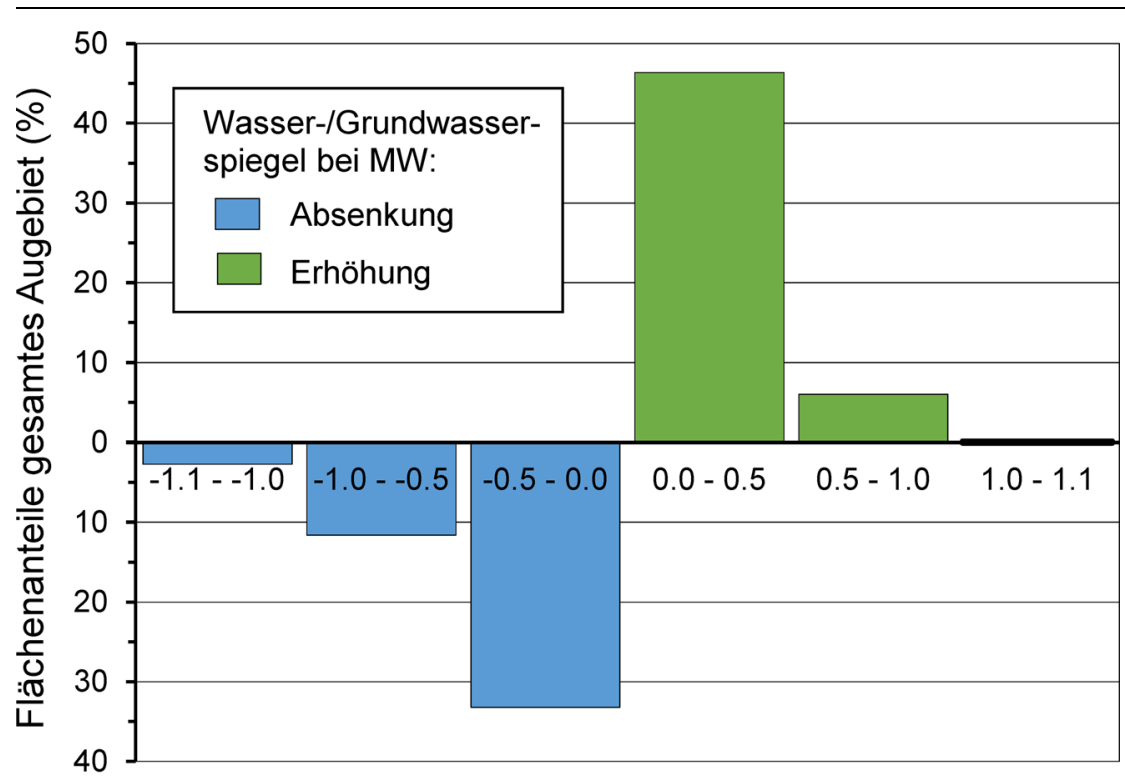

Absenkung bzw. Erhöhung (m)

Abb. 7 Absenkung und Erhöhung des Wasser- bzw. Grundwasserspiegels im östlichen Machland zwischen 1812 und 1817 infolge natürlicher Umlagerungsprozesse: Veränderungen der Spiegellagen in Halbmeterklassen und davon betroffene Flächenanteile des gesamten postglazialen Augebietes (\%)

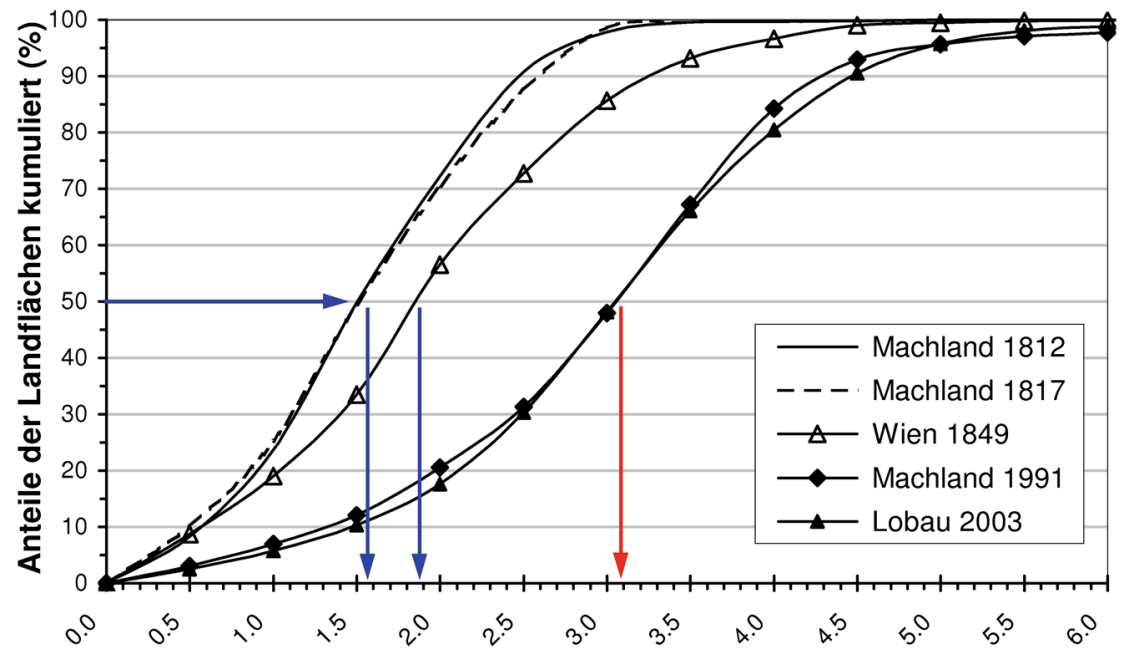

Flurabstand bei MW (m)

Abb. 8 Flächenanteile des Augeländes im Augebiet der Neuzeit (AZ) und zugehörige Flurabstände bei Mittelwasser (MW) dargestellt als Summenlinien: Vergleich Machland und Wien bzw. Lobau vor und nach Regulierung (die Pfeile zeigen die Medianwerte an; verändert nach Hohensinner et al. 2008, mit Genehmigung von Elsevier)

Spiegellagen von nur $0,04 \mathrm{~m})$. Trotz der umfassenden Veränderung der Flusslandschaft ist daher von einem annähernden Gleichgewichtszustand bezüglich der Spiegellagen und Flurabstände innerhalb des Gesamtsystems auszugehen. Dies ist auch anhand der Summenlinien in Abb. 8 belegbar, da der Medianwert (50-\%-Wert) der Flur- auf rund $3 \mathrm{~m}$ zu und verdoppelte sich damit annähernd (ca. 3,1 m im gesamten postglazialen Augebiet). Ebenso nahmen auch die Flurabstände in der Lobau bei Wien auf 3,1 m zu (der aktuelle Wert bezieht sich auf die Lob$\mathrm{au}$, da die Flurabstände im heutigen Wiener Stadtgebiet infolge des Stauraumes Freudenau und des umfassenden Grundwassermanagements wenig aussagekräftig sind). Somit haben sich innerhalb der letzten beiden Jahrhunderte neben den aquatischen Habitaten auch die Lebensraumbedingungen für die Auenvegetation im Hinblick auf den Grundwasseranschluss signifikant verändert.

\subsection{Wassertiefen und Sohllagen}

Wenn auch viele historische Vermessungen der Donau zum Zweck der Verbesserung der Schifffahrtsbedingungen durchgeführt wurden, so ist es doch verwunderlich, dass konkrete Informationen bezüglich der Sohllagen und Wassertiefen schwer zu finden sind. Grund dafür ist, dass meist nur Sondierungen entlang des Stromstrichs, der im Wesentlichen auch der Naufahrtslinie der talfahrenden Schiffe entsprach, vorgenommen wurden. So können meist nur Angaben zur Wassertiefe entlang des Stromstrichs gemacht werden, Aussagen über Flächenverteilungen von Wassertiefenzonen oder Vergleiche mit heutigen Sohllagen sind hingegen kaum möglich. Dabei erweist es sich wieder einmal als Glücksfall, dass der „Holler“ im Machland schon früh Gegenstand schifffahrtstechnischer Überlegungen war (Hohensinner 2010a). So wurden bereits 1817 und 1821 „Zick-Zack-Sondierungen" vorgenommen, indem man mit einem Boot von Ufer zu Ufer pendelte und dabei von der Strömung abgetrieben wurde. Auf Basis der damaligen Messwerte können grobe $\mathrm{Ge}$ ländemodelle erstellt werden, um so zu flächigen Aussagen bezüglich der Tiefenverhältnisse zu gelangen.

Demnach betrug die mittlere Tiefe der Hauptstromarme im Machland 1821 beim Nullwasserstand (ungefähr MJNW) auf die gesamte Wasserfläche bezogen rund $2 \mathrm{~m}$. Zum Vergleich für die aktuelle Situation sei hier wegen des heutigen Stauraumes nicht das Machland, sondern die freifließende Strecke im Bereich des Pilotprojektes Bad Deutsch-Altenburg vor Umsetzung der flussbaulichen Maßnahmen im Jahr 2006 herangezogen (Hohensinner und 


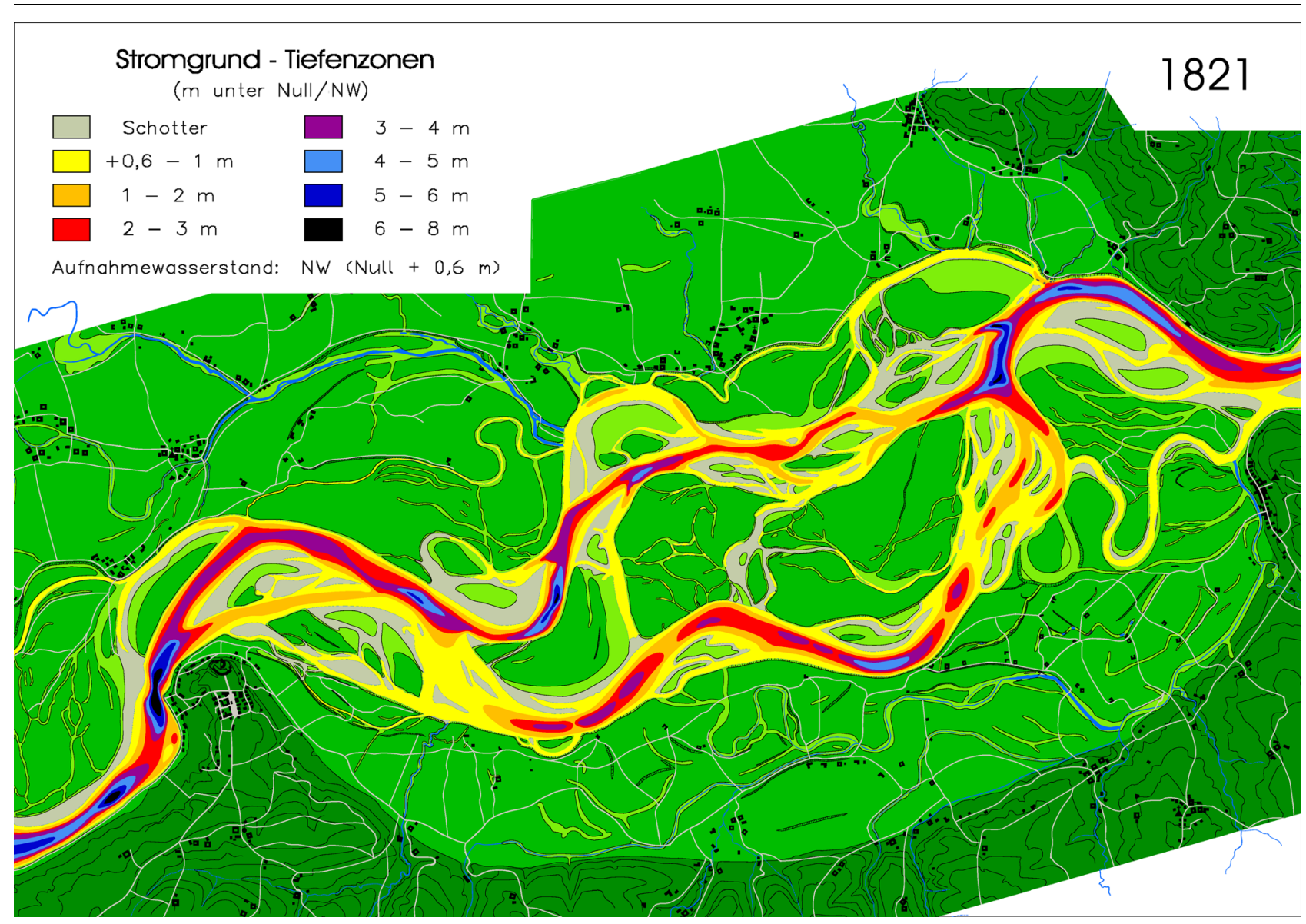

Abb. 9 Wassertiefenzonen derDonau imöstlichen Machland 1821 bezogenauf den Nullwasserspiegel (ungefähres MJNW; dieAusdehnung der Wasserflächen entspricht dem damaligen Aufnahmewasserstand von 0,6 m über Null; Hohensinner 2008)

Schuch 2010). Hier war der Hauptstrom mit 2,8 m bei RNW um $43 \%$ tiefer als in der Referenz vom Machland 1821. Bei Mittelwasser betrug der Unterschied sogar noch mehr: $2,6 \mathrm{~m}$ im Machland versus 3,9 m (+53\%) bei Bad Deutsch-Altenburg. Der Grund für die aktuell signifikant größere Wassertiefe ist sicherlich im wesentlich schmäleren Gerinneprofil der regulierten Donau $\mathrm{zu}$ finden. Aus fischökologischer Sicht besonders interessant sind Flachwasserzonen, da sie als Laich- und Aufwuchshabitate dienen. Bei Niederwasser nahmen solche Habitate mit bis $\mathrm{zu}$ einem Meter Tiefe ehemals $27 \%$ der Hauptstromwasserfläche ein, während es im aktuellen Vergleich lediglich rund $11 \%$ sind. Bei steigenden Wasserständen verringerten sich Flachwasserhabitate prozentuell auf die gesamte Wasserfläche bezogen aufgrund der starken Expansion des Hauptstromes (bzw. der Hauptstromarme), obwohl sie absolut gesehen eigentlich zunahmen. Bei Mittelwasser nahm die Zone mit bis zu einem Meter Wassertiefe im Jahr 1821 noch $20 \%$ ein, beim sommerlichen Mittelwasser hingegen nur mehr $13 \%$. Im Vergleich dazu erreichte diese Tiefenzone bei Bad Deutsch-Altenburg 2006 bezogen auf SMW lediglich 3,6\%. Dabei ist bei den aktuellen Werte noch zu bedenken, dass die Ausdehnung der Flachwasserhabitate aufgrund der wesentlich geringeren Gesamtwasserfläche absolut gesehen noch erheblich geringer ist als im ursprünglichen $\mathrm{Zu}$ stand vor der Regulierung. Konkret machten diese ehemals bei SMW ca. 8,1 ha pro km Luftlinie aus, während sie aktuell bei 1,2 ha pro $\mathrm{km}$ Luftlinie liegen, was einem Rückgang um $86 \%$ entspricht. Dabei ist noch nicht einmal berücksichtigt, dass es ehemals auch zahlreiche durchströmte Nebenarme mit vergleichbaren Habitaten gab, während diese heute nicht mehr existieren.

Auch die Sohlstrukturierung war ehemals stärker ausgeprägt als heute. So gab es im ehemals sehr breiten Hauptstrom Furten, die beim Null- wasserspiegel sogar weniger als einen Meter tief waren (vgl. gelb dargestellte Tiefenzonen in Abb. 9; Roidtner 1859). Das bedeutet, dass man damals die Hauptstromarme bei einer ausgeprägten Niederwassersituation theoretisch an besonders seichten Stellen zu Pferd überqueren konnte (tatsächlich war dies vermutlich aufgrund der dennoch starken Strömung nicht möglich).

Die historischen Wassertiefen der Neben- und Altarme wurden naturgemäß noch schlechter dokumentiert als die des Hauptstromes. In der Aufnahme der niederösterreichischen Donau von Lorenzo 1816 bis 1817 sowie in einigen anderen Kartenwerken finden sich jedoch Sondierungen, auf deren Basis für die Wiener Lobau - wenn auch mit Unsicherheiten behaftet eine gesonderte Auswertung der Tiefenverhältnisse je Gewässertyp für RNQ, MQ und bordvollem Abfluss (ca. HQ vorgenommen werden konnte. Auszugsweise werden hier die Ergebnisse bei Mittelwasser angeführt; bezüglich 


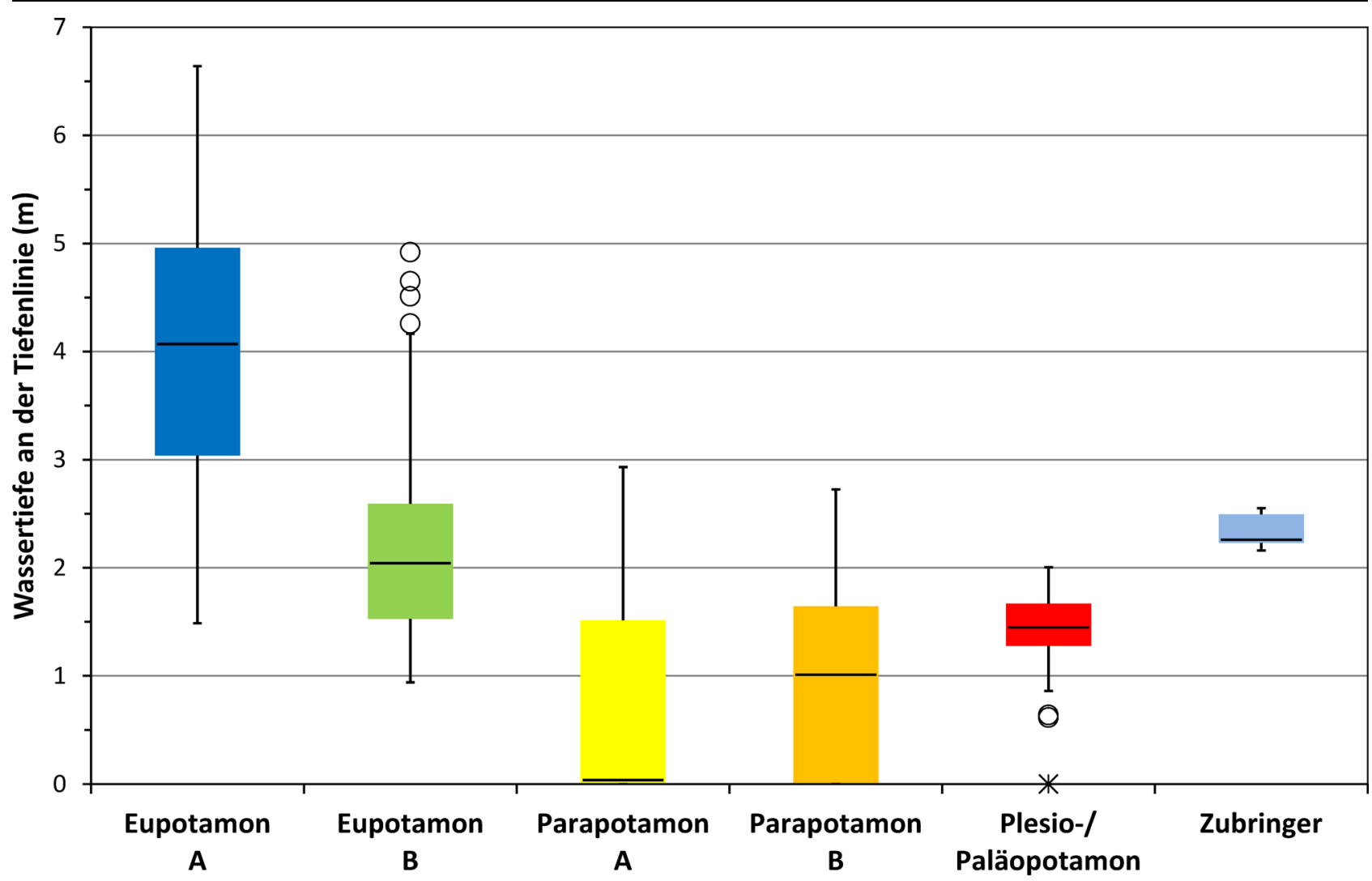

Abb. 10 Verteilung der Wassertiefen je Gewässertyp bei Mittelwasser in der Lobau 1817 (Werte bezogen auf die Tiefenlinie/Thalweg der Gewässer; Eupotamon A: Hauptstrom(arme), Eupotamon B: permanent durchströmte Nebenarme, Parapotamon A: zeitweise durchströmte dynamische Altarme, Parapotamon B: einseitig angebundene Altarme, Plesio-/Paläopotamon: Totarme; Querstriche kennzeichnen die Medianwerte; verändert nach Hohensinner und Trautwein 2013)

der Wassertiefen bei RNQ und $\mathrm{HQ}_{1}$ sei auf den Bericht Hohensinner und Trautwein (2013) verwiesen. Abgesehen von den Hauptstromarmen (Gewässertyp Eupotamon A) waren naturgemäß ständig durchströmte Nebenarme (Eupotamon B) entlang ihrer Tiefenlinie (Thalweg) gemessen mit rund $2 \mathrm{~m}$ im Median (50-\%-Wert) am tiefsten ausgebildet (Abb. 10). Überraschend erscheint, dass dynamische Altarme vom Typ Parapotamon A, die zumeist erst bei MW oder SMW durchströmt wurden, bei allen Wasserführungen nicht nur die geringsten Tiefen aufwiesen, die Verteilung der Tiefen war auch sehr asymmetrisch. Der Grund dafür ist, dass es sich hierbei um sehr heterogene Übergangstypen zwischen durchströmten Nebenarmen und anderen Altarmtypen handelt, die bei Niederwasser und zum Teil auch bei Mittelwasser durch große trockenfallende Abschnitte mit offenen Kiesflächen gekennzeichnet sind. Die Wassertiefe einseitig angebundener Altarme des Typs Parapotamon B betrug hingegen im Median bei MW ziemlich genau einen Meter. Totarme
(Plesio-/Paläopotamon) waren mit ca. 1,5 m entlang ihrer Tiefenlinie sogar noch etwas tiefer. Auffallend sind auch die vergleichsweise großen Tiefen der Zubringer (ehemalige Schwechat und Kalter Gang). Diese sind dadurch begründet, dass die kurzen Abschnitte im Projektgebiet meist vom Gewässersystem der Donau eingestaut wurden. Bei den dargestellten Werten handelt es sich naturgemäß um eine Momentaufnahme, wobei sich die Tiefen der einzelnen Augewässer ständig veränderten. Anzunehmen ist aber, dass solange sich die hydromorphologischen Rahmenbedingungen nicht signifikant änderten - ein dynamischer Gleichgewichtszustand herrschte, in dem die Verlandung bestehender Augewässer durch die Entstehung neuer Arme ausgeglichen wurde (vgl. Hohensinner et al. 2011).

Durch die Regulierungen im 19. Jahrhundert wurden solche ausgleichenden Entwicklungen jedoch immer mehr unterbunden. Dies spiegelt sich auch am Beispiel der Sohllagen wider, die sich in vergleichsweise kurzen Zeiträumen stark eintieften. Als Folge des Durchstichs durch den Weidenhaufen im Machland 1832 kam es flussaufwärts zu einer Eintiefung um rund 1,5 m. Diese Eintiefung wurde im Zuge weiterer regulierungs- und kraftwerksbedingter Maßnahmen zusätzlich verstärkt. Bereichsweise summierten sich die verschiedenen Phasen der Sohleintiefung im östlichen Machland schließlich auf insgesamt rund $2,7 \mathrm{~m}$. Wäre die Donau heute nicht eingestaut, würde der Wasserspiegel heute vermutlich um diesen Wert tiefer liegen als dies noch zwischen 1812 und 1821 der Fall war.

\subsection{Umlagerungsdynamik}

Wie bereits im Kapitel 3.1. zur generellen flussmorphologischen Charakteristik erwähnt, wird für Fluss-AuenSysteme des Typs der österreichischen Donau postuliert, dass sich die Sedimentations- und Erosionsprozesse über längere Zeiträume hinweg in einem dynamischen Gleichgewicht befinden (Nanson und Croke 1992). Die historischen Grundlagen lassen zwar 


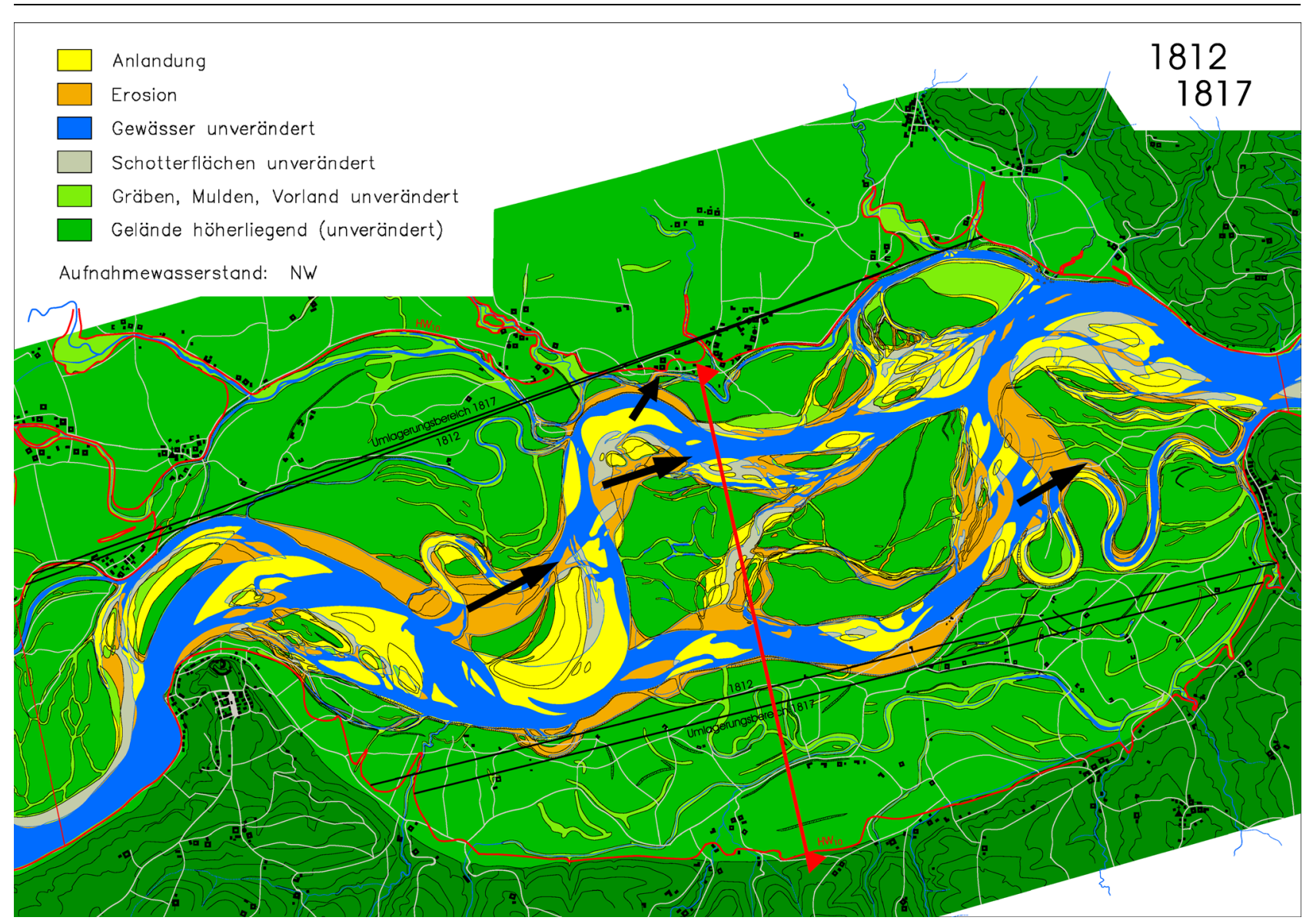

Abb. 11 Umlagerungen im östlichen Machland zwischen 1812 und 1817 (gelb: Anlandung, orange: Erosion, schwarze Pfeile: avulsive Durchbrüche, die Lage des in Abb. 12 dargestellten Transektes ist durch die rote Linie gekennzeichnet; Hohensinner 2008)

nur Analysen für einen vergleichsweise kurzen Zeitraum vor der Regulierung $\mathrm{zu}$, die Ergebnisse für das Machland und die Lobau unterstützen jedoch diese Hypothese. In der Lobau variierten zwischen 1805 und 1817 die erodierten und angelandeten Flächenanteile des Augebietes stark. In Summe ergibt sich jedoch mit jeweils $1,6 \%$ des vegetationsbedeckten Augeländes pro Jahr eine ausgeglichene Bilanz (bezogen auf das Augebiet der Neuzeit AZ). Im östlichen Machland, das im Vergleich zur Lobau eine stärkere Dynamik aufwies, betrugen Anlandung und Erosion zwischen 1812 und 1821 pro Jahr jeweils rund 2,5 \% des damaligen Augeländes. Rein rechnerisch wäre somit das gesamte Augelände auf diese Weise in 40 Jahren vollständig erneuert worden; tatsächlich blieben aber größere Flächen unberührt, während andere Bereiche wiederholt umgelagert wurden.

Die Verschneidung der Geländemodelle der Flusslandschaft im Machland 1812, 1817 und 1821 gewährt interessante Einblicke in die umgelagerten
Kubaturen. Zwischen 1812 und 1817 wurden zwei ausgeprägte Flussbögen am nördlichen Hauptarm im Zuge eines avulsiven Prozesses von der Donau abgekürzt und damit der betreffende Arm etwas begradigt (Abb. 11; vgl. dazu Ausgangssituation 1812 in Abb. 3). Zeitgleich wurde der um 1812 sehr seichte südliche Donauarm zum neuen Hauptarm, indem sich dessen Sohle um beinahe 1,5 m eintiefte und das Profil aufweitete (Abb. 12). Im Zuge dessen wurde auch eine Mäanderschlinge des östlich gelegenen mäandrierenden $\mathrm{Ne}$ benarmes bei Ardagger Markt vom neuen Hauptarm abgetrennt. Dabei wurden im Mittel jährlich 3 Millionen $\mathrm{m}^{3}$ an Material erodiert und 2,6 Millionen $\mathrm{m}^{3}$ innerhalb des östlichen Machlandes wieder abgelagert. Rund 0,4 Millionen $\mathrm{m}^{3}$ wurden durchschnittlich jedes Jahr aus dem System ausgetragen und durch den Strudengau weiter flussabwärts transportiert. In Summe machten die Umlagerungsvolumina demnach auf einer $10 \mathrm{~km}$ langen Strecke rund 15 Millionen $\mathrm{m}^{3}$ an erodiertem Mate- rial, 13 Millionen $\mathrm{m}^{3}$ an wieder abgelagertem Material und rund 2 Millionen $\mathrm{m}^{3}$ an ausgetragenem Sedimenten aus (Hohensinner et al. 2014). Davon war vermutlich das meiste Kies, ein Teil davon aber auch Material geringerer Korngröße, da auch damals die oberen Lagen des älteren Augeländes von Feinsedimenten gebildet wurden. Die Dimension der umgelagerten Volumina ist umso erstaunlicher, wenn man bedenkt, dass der Geschiebetransport der Donau bei Wien vor 1850 im Mittel pro Jahr rund 0,5 Millionen $\mathrm{m}^{3}$ ausmachte (Schmautz et al. 2000). Es ist aber nicht anzunehmen, dass das Material aus dem östlichen Machland sofort bis nach Wien weitertransportiert wurde. Vielmehr ist davon auszugehen, dass die Transportkapazität in einigen Donauabschnitten flussab des Machlandes nicht dazu ausreichte, die erhöhte Sedimentfracht zur Gänze weiterzutransportieren. Wahrscheinlich wurde ein Teil des Materials zwischenzeitlich im breiten Tullner Becken abgelagert und später remobilisiert. Wie bereits 


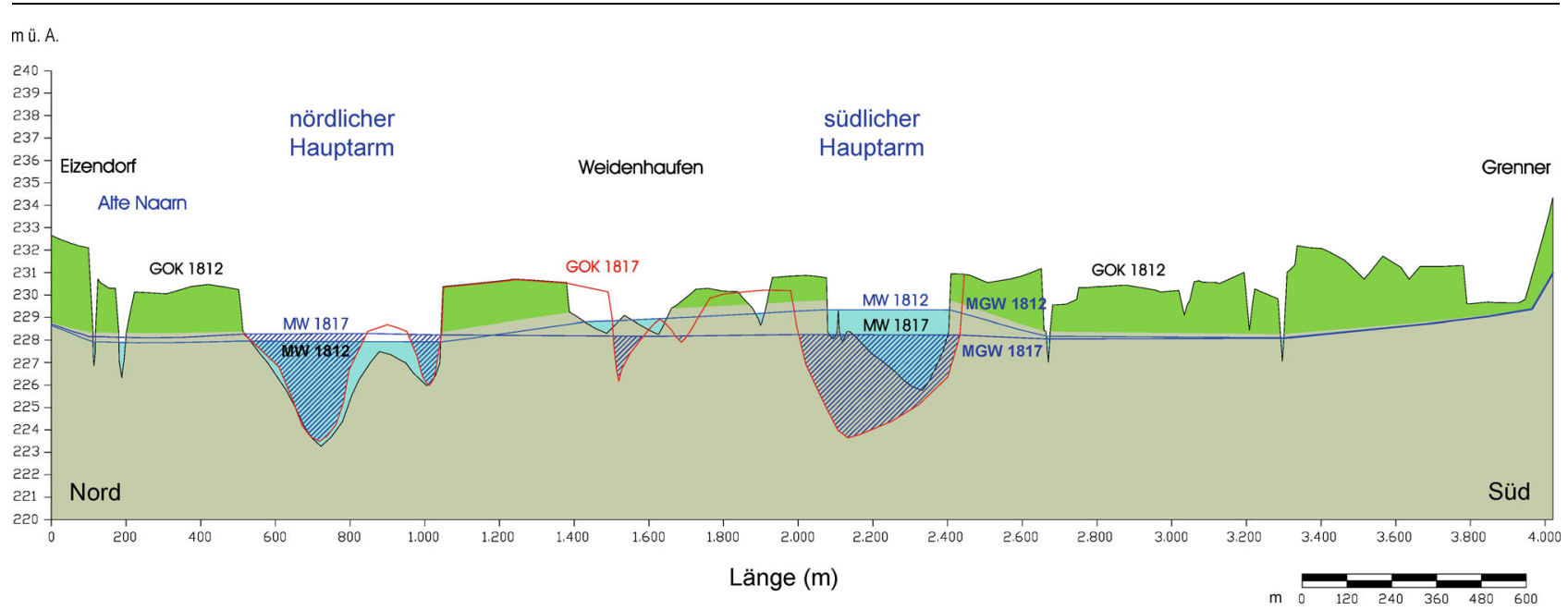

Abb. 12 Transekt durch die Flusslandschaft im östlichen Machland 1812 und Zustand 1817 überlagert (grün und braun: Gelände 1812, hellblau: Gewässer bei MW 1812, rot: Geländeoberkante 1817, dunkelblau schraffiert: Gewässer bei MW 1817; GOK Geländeoberkante, MW Mittelwasser, MGW mittlerer Grundwasserspiegel; verändert nach Hohensinner et al. 2007, 2014, mit Genehmigung von Elsevier)

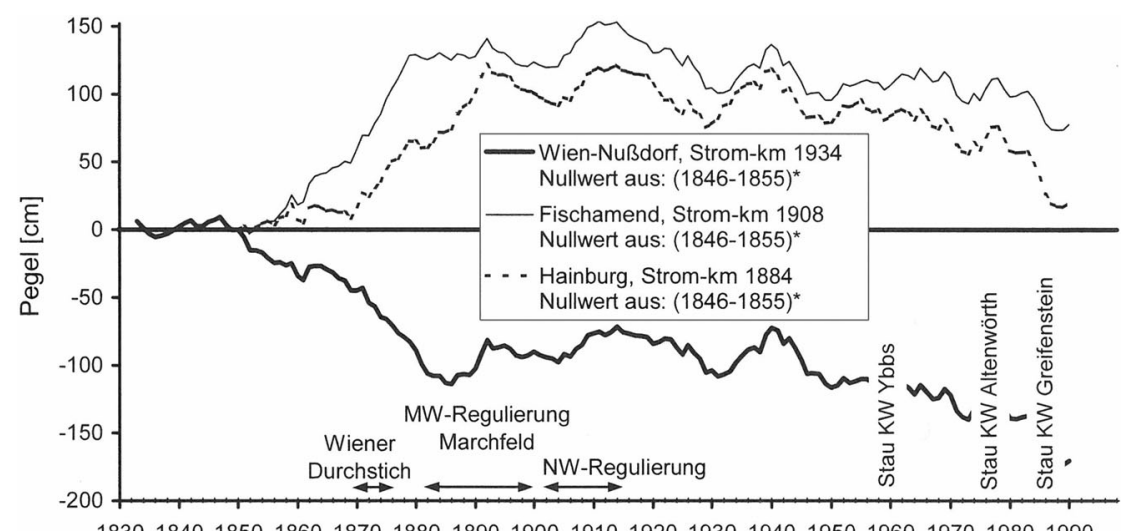

18301840185018601870188018901900191019201930194019501960197019801990 Jahr

Abb. 13 Entwicklung der Mittelwasserstände an der Donau in Wien und östlich von Wien seit 1830 dargestellt als gleitende Zehnjahresmittelwerte. ( $\left.{ }^{\star}\right)$ Mittelwerte der in Klammer angegebenen Zeiträume wurden als Referenz (Nullwerte) verwendet (aus Schmautz et al. 2002, mit freundl. Genehmigung durch die Autoren und den ÖIAV)

in Kapitel 3.3. näher ausgeführt, waren von den immensen morphologischen Veränderungen auch die Grundwasserspiegellagen bzw. Flurabstände in weiten Bereichen des Augebietes betroffen (vgl. Abb. 7 und MGW in Abb. 12).

Eine derart intensive Umlagerungsdynamik ereignete sich jedoch in ein und demselben Flussabschnitt nicht permanent. Der flussmorphologische Zustand der Donau im Machland 1812, gekennzeichnet durch eine hohe Sinuosität und zugleich starkes Gefälle des nördlichen Armes sowie Rückstau im Hauptstrom durch die Insel Weidenhaufen, deutete bereits darauf hin, dass größere Veränderungen zu erwarten waren (vgl. Abb. 3). In den darauffol- des 19. Jahrhunderts jedoch immer öfter. Grund dafür waren die zunehmenden Regulierungsarbeiten, wie zum Beispiel am beinahe $3 \mathrm{~km}$ langen Donau-Durchstich bei Markt Au in den 1820ern (ebenfalls im Machland) oder dem 2,3 km langen Durchstich am oben genannten Weidenhaufen 1832 . Da die Durchstiche zumeist durch hoch anstehendes Augelände verliefen und sich der Strom nach dem Aushub einer schmalen Initialkünette selbst den neuen Lauf ausschürfen musste, wurden dabei enorme Mengen an Sedimenten mobilisiert. Aufgrund der damit einhergehenden Laufverkürzung und Erhöhung des Gefälles kam es in weiterer Folge $\mathrm{zu}$ einer rückschreitenden Sohlerosion, wodurch weitere Geschiebemassen freigesetzt wurden. Ähnliches ist von den Regulierungsarbeiten am Unterlauf der Salzach und des Inns zwischen den 1860ern und 1880ern überliefert. Das dadurch mobilisierte Geschiebe verursachte in den folgenden Jahrzehnten eine Auflandung der Donausohle bei der Innmündung um rund 0,6 m, die sich im engen Donautal beim Jochensteiner Kachlet auf ca. $0,3 \mathrm{~m}$ reduzierte und ab da bis Aschach wieder zunahm (Ertl 1950). Das veränderte Sedimentregime ging damals mit größeren Veränderungen des Flussbetts einher.

Flussab von Wien, im heutigen Nationalpark Donau-Auen, beobachtete man ein anderes Phänomen. Seit den späten 1880ern lagerten sich vermehrt Sedimente in der Donau ab, wodurch riesige Kiesbänke und neue 
Inseln entstanden (Wiletal 1897). Dadurch ergaben sich neuerlich Probleme für die Schifffahrt. Als man im Jahr 1898 die Überreste des „Öden Schlosses" am linken Donauufer gegenüber Bad Deutsch-Altenburg untersuchte, stellte man überrascht fest, dass der gemauerte Fußboden nur $0,1 \mathrm{~m}$ bis $0,3 \mathrm{~m}$ über dem Mittelwasserstand lag (Genser 1989). Man schloss daraus, dass der Wasserspiegel der Donau ehemals erheblich tiefer gelegen sein musste. Untersucht man die Mittelwasserstände der Donau ab 1830 genauer, so werden die Auswirkungen der Regulierungsarbeiten auf die Höhenlage des Donauwasserspiegels offensichtlich. Während sich der Mittelwasserstand bei WienNussdorf zwischen 1850 und 1890 um mehr als einen Meter absenkte, stieg er $26 \mathrm{~km}$ stromabwärts bei Fischamend bis 1910 um 1,5 m an. In Hainburg, nochmals $24 \mathrm{~km}$ donauabwärts, betrug die Spiegelaufhöhung immerhin noch 1,2 m (Abb. 13; Schmautz et al. 2002; Klasz et al. 2016). Die Regulierungsarbeiten in der Donaustrecke flussauf des Nationalparks führten $\mathrm{zu}$ einer Eintiefung der Sohle, wodurch große Mengen an Sedimenten freigesetzt und weiter flussabwärts transportiert wurden. Östlich von Wien, wo das Gefälle der Donau vor der Regulierung mit 0,36 bis $0,40 \%$ vergleichsweise gering war, lagerte sich das mobilisierte Material teilweise wieder ab. Damit kam es sukzessive zu einer Erhöhung des Wasserspiegels. Um 1910 dürfte die Geschiebezufuhr aus der flussauf liegenden Strecke nachgelassen haben, wodurch sich der Trend spätestens ab den 1940ern umkehrte. Durch die darauf folgende Eintiefung des Flussbetts im 20. Jahrhundert sank der Mittelwasserstand wieder allmählich ab. Dieser Trend verstärkte sich in den letzten Jahrzehnten (Reckendorfer et al. 2005; Klasz et al. 2016). Man könnte nun annehmen, dass es einen Zeitpunkt gäbe, zu dem wieder ähnliche flussmorphologische Verhältnisse an der Donau östlich von Wien herrschen müssten, wie vor Beginn der Auflandung um 1850. Doch mittlerweile hat sich das Flusssystem stark verändert. Da über 100 Jahre hinweg ein höherer Wasserspiegel vorherrschte, war bei Hochwässern auch das Sedimentationsniveau im Augebiet höher. Demzufolge lagerten sich Feinsedimente im Augebiet in höheren Lagen ab, als dies vor der Regulierung der Fall gewesen war. Ebenso in Nebenund Altarmen, deren Sohlen auflande- ten (Jungwirth et al. 2014). Nach der starken Absenkung der Spiegellagen in den letzten Jahrzehnten ist nun der Höhenunterschied zwischen der Geländeoberkante im Augebiet bzw. der Sohllage der Altarme und dem aktuellen Mittelwasserspiegel der Donau größer als zuvor. Dies hat stark veränderte Standortsbedingungen für die Auenvegetation zur Folge: Die Au wurde trockener.

\section{Schlussfolgerung}

Aus Gründen der leichteren Datenverfügbarkeit wurden bisher im Rahmen von Restaurationsprojekten primär flussmorphologische Referenzwerte im Sinne eines Leitbildes herangezogen, welche die Ausprägung von Fließgewässersystemen anhand zweidimensionaler Lage-, Form- oder Flächenparameter beschreiben. Diese Vorgangsweise ist durchaus gerechtfertigt, da solche Informationen bezüglich der strukturellen Ausprägung von Gewässern in der Regel auch Aufschluss über die damit verbundenen ökologischen Funktionen geben. Dennoch lohnt es sich, gerade bei größeren Fluss-Auen-Systemen, die strukturellen Charakteristika und dynamischen hydromorphologischen Prozesse zusätzlich in vertikaler Dimension zu betrachten. Umso mehr, da sich unsere heutigen Fließgewässer und Auensysteme substanziell von ihrer Ausprägung vor der Regulierung unterscheiden. Ebenso können die Auswirkungen menschlicher Eingriffe umfassender und fundierter beurteilt werden, wenn auch die dritte Dimension berücksichtigt wird.

Im vorliegenden Artikel wurde der Beschreibung der Höhenlagen charakteristischer ökomorphologischer Geländezonen in Relation zum Niederwasser- oder Mittelwasserspiegel viel Platz eingeräumt. Dies kommt nicht von ungefähr, da verschiedene Bereiche eines Augebiets in Abhängigkeit von ihrer Lage zum Wasserbzw. Grundwasserspiegel auch unterschiedliche Funktionen innerhalb eines Ökosystems übernehmen - sei es als Habitat, bezüglich der Produktion von Biomasse oder als Ausgangs- oder Endpunkt von mobilisiertem Material (Sedimente, Totholz, Organismen). Höhere Geländelagen eines Augebiets stellen zumeist auch die älteren Habitate innerhalb eines Auensystems dar. Ältere Standorte wiederum zeichnen sich durch andere Charakteristika wie
Substratzusammensetzung, Bodenreife, Flurabstände, Überflutungshäufigkeit, -höhe und -dauer, Nährstoffgehalt, Chemismus etc. aus, als morphologisch jüngere. Jüngere und zumeist niedrigere Geländezonen - oft als Ökotone zwischen Wasser- und Landlebensräumen fungierend - spielen nicht nur als Standorte für Pioniervegetation eine wichtige Rolle, sie sind auch für die flussmorphologische Genese eines Gewässersystems besonders relevant: Hier sind die Auflandungsraten bei höheren Wasserständen besonders groß, wodurch sich wiederum die Standortsbedingungen und die Habitateignung für Organismen laufend ändern. Damit tragen sie besonders zum morphologisch heterogenen Mosaik zeitlich unterschiedlich entstandener Kompartimente des Gesamtsystems bei.

Die dreidimensionale Betrachtung der Donauflusslandschaft vor der Regulierung - strukturell sowie bezüglich ihrer hydromorphologischen Dynamik - verdeutlicht die intensiven Wechselbeziehungen und Austauschprozesse zwischen Wasser- und Landlebensräumen. Die terrestrischen Habitate eines Fluss-Auen-Systems waren vom schwankenden Abfluss und strukturellen Veränderungen des Gerinnesystems in verschiedenster Weise betroffen. Einerseits direkt, indem Geländeteile erodiert wurden oder durch Ablagerungen neu entstanden, zumeist aber - wie das Beispiel Machland zwischen 1812 und 1817 zeigt - indirekt, indem sich die Umlagerungen im Gewässersystem in signifikant geänderten hydrologischen Standortsbedingungen niederschlugen. Davon waren nicht nur die unmittelbar an den betreffenden Gewässern gelegenen Standorte betroffen, sondern auch weit davon entfernte Bereiche des $\mathrm{Au}$ gebiets. Wenn infolge einer avulsiven Verkürzung eines Flussbogens oder einer Mäanderschlinge schlagartig der Grundwasserspiegel um einen Meter oder sogar mehr absank, dann bedeutete dies, dass seichte Altarme und semiaquatische Feuchtlebensräume plötzlich trocken fielen und terrestrische Standorte wesentlich trockeneren Bedingungen unterlagen. Damit hätte sich der Charakter eines Fluss-Auen-Systems signifikant geändert. Das Beispiel aus dem Machland zeigt aber auch, dass zeitgleich andere Bereiche des Augebietes eine gegenläufige Entwicklung erfuhren, indem sich der Grundwasserspiegel merklich erhöhte. In Summe hielten sich Absenkung und Erhöhung 
der Wasser- und Grundwasserspiegellagen annähernd die Waage. Ein solcher Zustand lässt sich am besten mit dem viel zitierten „dynamischen Gleichgewicht“ beschreiben (zumindest aber weist das Beispiel aus dem Machland auf ein temporäres Gleichgewicht, eine Quasistabilität, hin; Hohensinner et al. 2011, 2014). In einem so dynamischen Ökosystem wie dem der Donau vor der Regulierung war es somit leicht möglich, dass Individuen, die z. B. einen bestimmten Feuchtlebensraum bewohnten, plötzlich gezwungen waren, sich ein neues Habitat zu suchen. $\mathrm{Da}$ aber zeitgleich innerhalb desselben Systems neue adäquate Habitate entstanden, wurden die davon betroffenen Populationen nicht nachhaltig beeinträchtigt. Ein solcher Gleichgewichtszustand existiert innerhalb eines Fließgewässersystems jedoch nur, solange sich die äußeren Rahmenbedingungen wie das Klima und damit zusammenhängend das Abflussregime nicht signifikant verändern. Dies trifft ebenfalls auf das Sedimentregime zu, das zum Teil auch klimatisch bedingt ist. Massive temporäre Geschiebeeinträge wie im Machland 1812 bis 1817 mögen zwar kurzfristig die Transportkapazität der Donau überragen und zu verstärkten Ablagerungen im nächsten Augebiet flussab davon führen, nach der Freisetzung des Materials aber folgten mehrere Jahre der morphologischen Konsolidierung. Das 1817 viel zu ausgedehnte aktive Gerinnesystem nahm nunmehr zum Ausgleich angeliefertes Material aus der Oberliegerstrecke auf. So ergab sich entlang des Donaulaufes ein dynamisches Kontinuum der Sedimentmobilisierung einerseits und -fixierung innerhalb der Auenkomplexe andererseits.

Nicht nur an der Donau, sondern entlang der meisten größeren Flüsse weisen die Augebiete heute wesentlich höhere Geländelagen in Relation zum Wasser- und Grundwasserspiegel auf, als dies vor der Regulierung der Fall war. Sohleintiefung als Folge eines stark reduzierten oder gar fehlenden Geschiebetransportes, verbunden mit der Einengung der Gerinneprofi- le im Zuge der Regulierung, ist dabei als Hauptursache anzusehen. Verstärkt wird dieses Problem jedoch noch durch die stetige Ablagerung von Feinsedimenten bei Hochwässern im Augebiet. Die Untersuchungen im östlichen Machland zeigen, dass sich dadurch die Geländeoberkante zwischen 1812 und 1991 bereichsweise um bis $\mathrm{zu} 1,5 \mathrm{~m}$ erhöht hat. In diesem Zeitraum wurden rund 34 Millionen $\mathrm{m}^{3}$ an Material abgelagert. Im Gegenzug wurden ca. 20 Millionen $\mathrm{m}^{3}$ Sedimente erodiert, wobei „erodiert“ in diesem Zusammenhang ein missverständlicher Ausdruck ist. Denn dieses Volumen resultiert primär aus der Begradigung des Donaulaufes und der Eintiefung der Sohle. Nicht enthalten sind die mittlerweile bei den Hochwässern 2002 und 2013 abgelagerten Feinsedimente, welche an einigen Stellen Mächtigkeiten von 1 bis $2 \mathrm{~m}$ erreichten und die mancherorts zur Einebnung von Gräben und verlandenden Kleingewässern verwendet wurden. Absinkende Wasser- bzw. Grundwasserspiegel einerseits sowie sich stetig erhöhende Augebiete andererseits führen zu einer immer stärkeren vertikalen Entkoppelung der Wasser- und Landlebensräume der Fluss-Auen-Systeme. Die Flurabstände vergrößern sich und die ehemals nassen, feuchten oder frischen Standorte der Weichen Au gehen sukzessive verloren. Da als Folge fehlender Dynamik kaum mehr neue Habitate entstehen, unterliegen die seit der Regulierung stabilisierten Bereiche einem gleichförmigen Alterungsprozess, indem sich ihre abiotischen Charakteristika aber auch ihre ökologischen Funktionen immer stärker angleichen (Hohensinner et al. 2011).

Die hier beschriebenen Beispiele aus der Geschichte der österreichischen Donau zeigen, dass eine flusstypische - und im Falle der Donau hohe - Umlagerungsintensität, durch die große Anteile der aquatischen und terrestrischen Habitate in vergleichsweise kurzen Zeiträumen regeneriert und verjüngt wurden, als Schlüssel zur Aufrechterhaltung eines heterogenen Habitatkomplexes anzusehen ist. Folglich erweisen sich zwei Aspekte für eine nachhaltige Restauration von Fluss-Auen-Systemen als essenziell: ausreichend Raum und genug Geschiebeinput für ein dynamisches Prozessgeschehen.

\section{Danksagung}

Der vorliegende Artikel beruht auf Ergebnissen zahlreicher Forschungsprojekte, wofür folgenden Fördergebern beziehungsweise Auftraggebern an dieser Stelle herzlich gedankt sei: dem Fonds zur Förderung der wissenschaftlichen Forschung (FWF-Projekte „Machland 1715 - 1991“, Nr. P14959-B06; „DYNAFISH - Historische Entwicklung von Fischgemeinschaften“, Nr. I450B17; „ENVIEDAN - Umweltgeschichte der Wiener Donau 1500 - 1890“, Nr. P22265-G18; „URBWATER - Vienna's Urban Waterscape 1683 - 1918", Nr. P25796-G18); dem Jubiläumsfonds der Stadt Wien für die Österreichische Akademie der Wissenschaften (Projekt „Genug Holz für Stadt und Fluss? Wiens Holzressourcen in dynamischen Donau-Auen"); dem Bundesministerium für Wissenschaft, Forschung und Wirtschaft (proVISION-Projekt „Optima Lobau“, EZ 133-260/05); der MA 45 Wiener Gewässer bzw. WasserCluster Lunz - Biologische Station GmbH (Projekt „Hydrodynamische Charakteristik aquatischer Habitate in der Lobau um 1817“) und der via donau - Österreichische Wasserstraßen-Gesellschaft $m b H$ (Premonitoring „Pilotprojekt Bad Deutsch-Altenburg“).

Open access funding provided by University of Natural Resources and Life Sciences Vienna (BOKU).

Open Access Dieser Artikel wird unter der Creative Commons Namensnennung 4.0 International Lizenz (http:// creativecommons.org/licenses/by/4. 0/deed.de) veröffentlicht, welche die Nutzung, Vervielfältigung, Bearbeitung, Verbreitung und Wiedergabe in jeglichem Medium und Format erlaubt, sofern Sie den/die ursprünglichen $\mathrm{Au}$ tor(en) und die Quelle ordnungsgemäß nennen, einen Link zur Creative Commons Lizenz beifügen und angeben, $\mathrm{ob}$ Änderungen vorgenommen wurden. 
Chovanec, A., Waringer, J., Straif, M., Graf, W., Reckendorfer, W., Waringer-Löschenkohl, A. Waidbacher, H. \& Schultz, H. (2005): The Floodplain Index - a new approach for assessing the ecological status of river/floodplain-systems according to the EU Water Framework Directive. Archiv für Hydrobiologie Supplementband, 155, Large Rivers, 15 (1-4), 169-185.

de Lorenzo, C. (1819): Nieder Oesterreichische Donau-Stromkarte, M: $1: 7.200$, aufgenommen 1816 - 1817, hrsg. 1819, 69 Bl., NÖLB B II 82. Desloges, J.R. \& Church, M. (1987): Channel and floodplain facies in a wandering gravel-bed river. In: Ethridge, F.G., Flores, R.M. \& Harvey, M.D. (Hrsg.), Recent Developments in Fluvial Sedimentology. Special Publication Number 39. Society of Economic Paleontologists and Mineralogists, Tulsa, Oklahoma, S. 99-109.

Doyle, M.W., Shields, D., Boyd, K.F., Skidmore, P.B. \& Dominick, D. (2007): Channel-forming discharge selection in river restoration design. Journal of Hydraulic Engineering, 133, 831-837.

Drescher, A. \& Egger, G. (2013): Wiener Holz - Vegetation. Bericht für das Projekt „Genug Holz für Stadt und Fluss? Wiens Holzressourcen in dynamischen Donau-Auen“ im Auftrag des Instituts für Hydrobiologie \& Gewässermanagement, BOKU Wien, Karl-Franzens-Universität Graz und eb \& p Umweltbüro GmbH Klagenfurt. Drescher, A., Egger, G., Haidvogl, G. \& Hohensinner, S. (submitted): Reconstructing the riparian vegetation prior to regulation: the Viennese Danube floodplain in 1825. Limnologica. Ertl (1950): Die Gestaltungsvorgänge am Inn unterhalb der Salzachmündung. Gutachten, München.

EU - Europäisches Parlament (2000): Richtlinie 2000/60/EG zur Schaffung eines Ordnungsrahmens für Maßnahmen der Gemeinschaft im Bereich der Wasserpolitik hat die Umweltpolitik der Europäischen Gemeinschaften.

Friderich (Friedrich) (1812/13): Aufnahme des Donaustromes von dem Markte Hütting und Wallsee über Dorna und Ardacker bis zur Donau=Enge - dem sogenannten Saurüsl“, aufgenommen 1812, hrsg. 1813, OÖLA StromkartenMappe Nr. 1.

Genser, K. (1989): Der österreichische Donaulimes in der Römerzeit. Ein Forschungsbericht Der römische Limes in Österreich, 33, S. 662.

Graf, W., Chovanec, A., Hohensinner, S., Leitner, P., Schmidt-Kloiber, A., Stubauer, I., Waringer, J. \& Ofenböck, G. (2013): Das Makrozoobenthos als Indikatorgruppe zur Bewertung großer Flüsse unter Einbeziehung auenökologischer Aspekte. Österreichische Wasser- u. Abfallwirtschaft, 65, 386-399.

Grupe, S. \& Payer, T. (2014): Simmering. Vortragsreihe Hydrogeologie von Wien - Teilgebiet 2014. Wiener Gewässer Management, 28. 11. 2014.

Haidvogl, G. \& Eberstaller, J. (2010): LIFE+ Lebensraum im Mündungsabschnitt des Flusses Traisen. Umweltverträglichkeitserklärung. Fachbeitrag Synthese Pflanzen, Tiere, Lebensräume. Bericht im Auftrag von Verbund - Austrian Hydro Power AG, $98 \mathrm{~S}$

Hein, T., Lazowski, W., Muhar, S., Schwarz, U. \& Weigelhofer, G. (2014): Die Bedeutung von Augewässern am Beispiel der Donau-Auen bei Wien. Denisia, 33, 167-174.

Herrnegger, M. (2007): Historische Hydromorphologie und Geländetopografie der Wiener Donau-Auen; Diplomarbeit am Institut für Hydrobiologie und Gewässermanagement, Universität für Bodenkultur Wien, 142 S. mit Kartenbeilagen.
Hohensinner, S. (1995): Bilanzierung historischer Flussstrukturen im Oberen Donautal als Grundlage für die Revitalisierung des ehemaligen Altarmes bei Oberranna. Diplomarbeit an der Universität für Bodenkultur Wien, 179 S.

Hohensinner, S. (2008): Rekonstruktion ursprünglicher Lebensraumverhältnisse der FlussAuen-Biozönose der Donau im Machland auf Basis der morphologischen Entwicklung von 1715 - 1991. Dissertation an der Universität für Bodenkultur Wien, 307 S., 27 Karten.

Hohensinner, S. (2010a): „Wie viele Fahrzeuge liegen in den Schottermassen begraben?“ Die Schifffahrt auf der unregulierten Donau. In: Winiwarter, V. \& Schmid, M. (Hrsg.), Umwelt Donau: Eine andere Geschichte. Katalog zur Ausstellung des NÖ Landesarchivs „Donau - Fluch \& Segen“ in Ardagger Markt, 5. Mai 7. Nov. 2010, NÖ Institut für Landeskunde, St. Pölten, 104-117.

Hohensinner, S. (2010b): „Sobald jedoch der Strom einen anderen Lauf nimmt ..." - Der Wandel der Donau vom 18. zum 20. Jahrhundert. In: Winiwarter, V. \& Schmid, M. (Hrsg.), Umwelt Donau: Eine andere Geschichte. Katalog zur Ausstellung des NÖ Landesarchivs „Donau - Fluch \& Segen“ in Ardagger Markt, 5. Mai - 7. Nov. 2010, NÖ Institut für Landeskunde, St. Pölten, 38-55.

Hohensinner, S. \& Jungwirth, M. (2009): Flussmorphologische Charakteristik der Donau - historische Perspektive. Österreichische Ingenieuru. Architekten-Zeitschrift, 154 (1-6), 33-38.

Hohensinner, S. \& Schuch, M. (2010): Naturversuch Bad Deutsch-Altenburg - Premonitoring End-bericht, Arbeitspaket B2b Landschaftsdynamik/Leitbild. In: Schiemer, F., Habersack, H. \& Schabuss, M. (Hrsg.), Endbericht im Auftrag der via donau - Österreichische WasserstraßenGesellschaft mbH.

Hohensinner, S. \& Trautwein, C. (2013): Hydrodynamische Charakteristik aquatischer Habitate in der Lobau um 1817. Projektbericht im Auftrag von MA 45 - Wiener Gewässer u. WasserCluster Lunz, Institut für Hydrobiologie \& Gewässermanagement, Universität für Bodenkultur Wien, $32 \mathrm{~S}$

Hohensinner, S., Habersack, H., Jungwirth, M. \& Zauner, G. (2004): Reconstruction of the characteristics of a natural alluvial river-floodplain system and hydromorphological changes following human modifications: the Danube River (1812-1991). River Research and Applications, 20 (1), 25-41.

Hohensinner, S., Jungwirth, M., Muhar, S. \& Habersack, H. (2005): Historical analyses: a foundation for developing and evaluating rivertype specific restoration programs. International Journal of River Basin Management, 3 (2), 87-96. Hohensinner, S., Egger, G., Haidvogl, G., Jungwirth, M., Muhar, S. \& Schmutz, S. (2007): Hydrological connectivity of a Danube riverfloodplain system in the Austrian Machland: changes between 1812 and 1991. In: Trémolières, M., Schnitzler, A. \& Silan, P. (Hrsg.), Floodplain Protection, Restoration, Management. Why and how? Editions Lavoisier SAS, Paris, 53-69.

Hohensinner, S., Herrnegger, M., Blaschke, A.P. Habereder, C., Haidvogl, G., Hein, T., Jungwirth, M. \& Weiß, M. (2008): Type-specific reference conditions of fluvial landscapes: a search in the past by 3D-reconstruction. Catena, 75, 200-215. Hohensinner, S., Jungwirth, M., Muhar, S. \& Schmutz, S. (2011): Spatio-temporal habitat dynamics in a changing Danube River landscape
1812 - 2006. River Research and Applications, 27, 939-955.

Hohensinner, S., Sonnlechner, C., Schmid, M. \& Winiwarter, V. (2013a): Two steps back, one step forward: reconstructing the dynamic Danube riverscape under human influence in Vienna. Water History, 5 (2), 121-143.

Hohensinner, S., Lager, B., Sonnlechner, C., Haidvogl, G., Gierlinger, S., Schmid, M., Krausmann, F. \& Winiwarter, V. (2013b): Changes in water and land: the reconstructed Viennese riverscape 1500 to the present. Water History, 5 (2), 145-172.

Hohensinner, S., Drescher, A., Eckmüllner, O., Egger, G., Gierlinger, S., Hager, H., Haidvogl, G. \& Jungwirth, M. (2013c): Genug Holz für Stadt und Fluss? Wiens Holzressourcen in dynamischen Donau-Auen. Projektbericht, Universität für Bodenkultur Wien, 66 S. mit 12 Kartenbeilagen.

Hohensinner, S., Jungwirth, M., Muhar, S. \& Schmutz, S. (2014): Importance of multi-dimensional morphodynamics for habitat evolution: Danube River 1715 - 2006. Geomorphology, 215, 3-19.

Höchsmann, A. (1848): Hydrotechnischer Plan der Donaustrecke von Nieder Wallsee bis Ardagger zum Behufe der Strom Regulierung mit Bezug auf das beigelegte Programm. M: 1 : 14.400, Planungsoperat mit Bezug zur Situation um ca. 1831, M:

1 : 14.400, Privatarchiv Christoph Matschnig in Wien sowie Herrschaftsarchiv Wallsee.

HZB - Hydrographisches Zentralbüro (1937): Schwebstoff- und Geschiebeaufnahmen einiger österreichischer Flüsse. Mitteilungen des Hydrographischen Zentralbüros, Heft 51.

Jungwirth, M., Eberstaller, J., Haidvogl, G., Hohensinner, S., Küblbäck, G., Pinka, P. \& Schmalfuß, R. (2005): Machbarkeitsstudie „Rückbau Traisenunterlauf und Traisenmündung“. Bericht im Auftrag von Amt der Nö. Landesregierung, Forstverwaltung Schloss Grafenegg, Augustiner Chorherrenstift Herzogenburg, Agrargemeinschaft Lehenteil und BMLFUW, $56 \mathrm{~S}$.

Jungwirth, M., Haidvogl, G., Hohensinner, S., Waidbacher, H. \& Zauner, G. (2014): Österreichs Donau. Landschaft - Fisch - Geschichte. Institut für Hydrobiologie u. Gewässermanagement, BOKU Wien, $420 \mathrm{~S}$.

Killian, K. (vermutlich 1970er): Lage- und Schichtenplan des Donaugeländes bei Wien 1849. Rekonstruktion basierend auf dem plastischen Modell von Streffleur, V., Kazda \& Drobny, C. (1849), welches mittels ca. 250 Höhenpunkten neu kalibriert wurde, Archiv der MA 29 Baugrundkataster.

Klasz, G., Reckendorfer, W. \& Gutknecht, D. (2012): Morphological aspects of bankfull and effective discharge of gravel-bed rivers and changes due to channelization. $9^{\text {th }}$ International Symposium on Ecohydraulics 2012, Proceedings.

Klasz, G., Gabriel, H., Habersack, H., Schmalfuß, R., Baumgartner, C. \& Gutknecht, D. (2016): Ausmaß und Dynamik der Sohlerosion der Donau östlich von Wien - flussmorphologische und wasserwirtschaftliche Aspekte. Österreichische Wasser- $u$. Abfallwirtschaft, DOI: 10.1007/s00506-016-0309-4.

Leopold, L.B. \& Wolman, M.G. (1957): River channel patterns: braided, meandering, and straight. U.S. Geological Survey Professional Paper 282-B, 39-85. 
Mitis, F., Ritter v. (1835): Geschichte des Wiener Donau-Canales und Darstellung der Ursachen seines unvollkommenen schiffbaren Zustandes. Wien.

Mohilla, P. \& Michlmayr, F. (1996): Donauatlas Wien. Geschichte der Donauregulierung auf Karten und Plänen aus vier Jahrhunderten. Österreichischer Kunst- und Kulturverlag, ca. 120 Bl., Wien.

Muhar, S., Pohl, G., Stelzhammer, M., Jungwirth, M., Hornich, R. \& Hohensinner, S. (2011): Integratives Flussgebietsmanagement: Abstimmung wasserwirtschaftlicher, gewässerökologischer und naturschutzfachlicher Anforderungen auf Basis verschiedener EU-Richtlinien (Beispiel Steirische Enns. Österreichische Wasser- u. Abfallwirtschaft, 9-10, 167-173.

Nanson, G.C. \& Croke, J.C. (1992): A genetic classification of floodplains. Geomorphology, 4, 459-486.

Nanson, G.C. \& Knighton, A.D. (1996): Anabranching rivers: their cause, character and classification. Earth Surface Processes and Landforms, 21, 217-239.

Natura 2000, Richtlinie 92/43/EWG des Rates vom 21. Mai 1992 zur Erhaltung der natürlichen Lebensräume sowie der wildlebenden Tiere und Pflanzen (Fauna-Flora-Habitat-Richtlinie).

Parker, G., Wilcock, P.R., Paola, C., Dietrich, W.E. \& Pitlick, J. (2007): Physical basis for quasiuniversal relations describing bankfull hydraulic geometry of single-thread gravel bed rivers. Journal of Geophysical Research, 112, F04005, doi:10.1029/2006JF000549, 21 S.

Pasetti, F., Ritter v. (1862): Notizen über die Donau-Regulirung im österreichischen Kaiserstaate bis zu Ende des Jahres 1861 mit Bezug auf die im k.k. Staatsministerium herausgegebene
Übersichts-Karte der Donau (Karte des DonauStromes, Theil 2). Wien, $39 \mathrm{~S}$

Powell G.E., Mecklenburg D. \& Ward A.D. (2006): Evaluation of Channel-Forming Discharges - A Study of Large Rivers in Ohio. American Society of Agricultural and Biological Engineers, 49, S. 35-46.

Reckendorfer, W., Schmalfuß, R., Baumgartner, C., Habersack, H., Hohensinner, S., Jungwirth. M. \& Schiemer, F. (2005): The Integrated River Engineering Project for the free-flowing Danube in the Austrian Alluvial Zone National Park: contradictory goals and mutual solutions. Archiv für Hydrobiologie Supplementband, 155, Large Rivers, 15 (1-4), 613-630.

Roidtner, J. (1859): Die Regulirung des DonauHollers im Strombecken oberhalb des Strompasses bei Grein. Allgemeine Bauzeitung, 9 (10) 244-252.

Schmalfuß, R. \& Eberstaller, J. (2010): LIFE+ Lebensraum im Mündungsabschnitt des Flusses Traisen. Umweltverträglichkeitserklärung. Zusammenfassende Darstellung. Bericht im Auftrag von Verbund - Austrian Hydro Power AG, 146 S.

Schmautz, M., Aufleger, M. \& Strobl, T. (2000): Wissenschaftliche Untersuchung der Geschiebe- und Eintiefungsproblematik der österreichischen Donau. Bericht im Auftrag von Verbund Austrian Hydro Power AG (AHP), Wien.

Schmautz, M., Aufleger, M. \& Strobl, T. (2002): Anthropogene Einflussnahme auf die Flussmorphologie der Donau in Österreich. Österreichische Ingenieur- u. Architekten-Zeitschrift, 147 (5-6), 171-178.

Streffleur, V. (1846/47): Tiefenwasser in der Donau bei Wien. Mittelwasser in der Donau be Wien. Hochwasser in der Donau bei Wien. Karten im Maßstab 1 : 28.800 vermutlich unter der Leitung von Valentin von Streffleur erstellt, Geologische Bundesanstalt K IV 2591-2593 Streffleur, V. (1851): Einiges über Wasserstands(Pegel-) Beobachtungen und deren Aufzeichnungen. Sitzungsberichte der Kaiserlichen Akademie der Wissenschaften. Mathematisch-naturwissenschaftliche Classe. Bd. 7, Heft 1, 745756.

Streffleur, V., Kazda \& Drobny, C. (1849): Hydrotechnische Vermessung der Donau bei Wien. M: 1 : 14.400, Originalaufnahme verschollen, stattdessen existiert heute noch ein plastisches Geländemodell mit einem Isohypsenabstand von 0,2 Klafter $(0,38 \mathrm{~m})$ von Carl Drobny, Depot des Technischen Museums Wien, Sign. L20800. Stummer, D. (1986): Veränderungen der Stromlandschaft im Stadtbereich von Wien seit 1780 an ausgewählten Kartenbeispielen. Unveröffentlichte Hausarbeit in Biologie, Innsbruck, Archiv der Geologischen Bundesanstalt in Wien, $55 \mathrm{~S}$. mit Kartenanhang.

Van den Berg, J.H. (1995): Prediction of alluvial channel pattern of perennial rivers. Geomorphology 12 (4), S. 259-279.

Wex, G. (1873): Ueber die Wasserabnahme in den Quellen, Flüssen und Strömen. Zeitschrift des österreichischen Ingenieurs- u. ArchitektenVereines, 25, 23-30, 63-76, 101-119.

Wiletal, I. (1897): Die Veränderungen des Donau-Laufes im Wiener Becken. Alt-Wien - Monatsschrift für Wiener Art und Sprache, 6, S. 4851, S. 65-68.

Wolman, M.G. \& Leopold, L.B. (1957): River flood plains - Some observation on their formation. U.S. Geological Survey Professional Paper 282-C, S. 87-109.

Yalin, M.S. \& da Silva, A.M.F. (2001): Fluvial Processes. IAHR Monograph, IAHR, Delft, The Netherlands. 